\title{
Homophily and long-run integration in social networks
}

\author{
Yann Bramoullé a,b, Sergio Currarini ${ }^{\mathrm{c}, \mathrm{d}}$, Matthew O. Jackson ${ }^{\mathrm{e}, \mathrm{f}, \mathrm{g}, *}$, \\ Paolo Pin ${ }^{\mathrm{h}}$, Brian W. Rogers ${ }^{\mathrm{i}, \mathrm{j}}$ \\ a Department of Economics, Université Laval, Canada \\ ${ }^{\mathrm{b}}$ Aix-Marseille School of Economics, France \\ ${ }^{\mathrm{c}}$ Università di Venezia, Italy \\ ${ }^{\mathrm{d}}$ University of Bristol, United Kingdom \\ e Department of Economics, Stanford University, CA, United States \\ f CIFAR, Canada \\ $\mathrm{g}$ The Santa Fe Institute, NM, United States \\ ${ }^{\mathrm{h}}$ Dipartimento di Economia Politica e Statistica, Università degli Studi di Siena, Italy \\ ${ }^{\mathrm{i}}$ MEDS, Kellogg School of Management, Northwestern University, IL, United States \\ j NICO, Northwestern University, IL, United States
}

Received 1 September 2010; final version received 21 January 2012; accepted 5 February 2012

\begin{abstract}
We model network formation when heterogeneous nodes enter sequentially and form connections through both random meetings and network-based search, but with type-dependent biases. We show that there is "long-run integration", whereby the composition of types in sufficiently old nodes' neighborhoods approaches the global type-distribution, provided that the network-based search is unbiased. However, younger nodes' connections still reflect the biased meetings process. We derive the type-based degree
\end{abstract}

\footnotetext{
Following the suggestion of JET editors, this paper draws from two working papers developed independently: Bramoullé and Rogers (2010) [4] and Currarini, Jackson, and Pin (2010) [11]. We gratefully acknowledge financial support from the NSF under grant SES-0961481 and we thank Vincent Boucher for his research assistance. We thank the staff from the American Physical Society (APS) Journal Information Systems (http://publish.aps.org/departments/ journalinformationsystems) for their assistance. We also thank Habiba Djebbari, Andrea Galeotti, Sanjeev Goyal, James Moody, Filippo Radicchi, Betsy Sinclair, Bruno Strulovici, and Adrien Vigier, as well as numerous seminar participants and the associate editor and anonymous referees for helpful comments and suggestions.

* Corresponding author at: Department of Economics, Stanford University, CA, United States.

E-mail addresses: s.currarini@unive.it (S. Currarini), jacksonm@stanford.edu (M.O. Jackson), paolo.pin@unisi.it (P. Pin), b-rogers@ northwestern.edu (B.W. Rogers).

URL: http://www.stanford.edu/ jacksonm/ (M.O. Jackson).
} 
distributions and group-level homophily patterns when there are two types and location-based biases. Finally, we illustrate aspects of the model with an empirical application to data on citations in physics journals. (C) 2012 Elsevier Inc. All rights reserved.

JEL classification: D85; A14; Z13

Keywords: Network formation; Social networks; Homophily; Integration; Degree distribution; Citations

\section{Introduction}

Homophily patterns in networks have important consequences. For example, citations across literatures can affect whether, and how quickly, ideas developed in one field diffuse into another. Homophily also affects a variety of behaviors and opportunities, with impact on the welfare of individuals connected in social networks. ${ }^{1}$ In this paper we analyze a model that provides new insights into the patterns and emergence of homophily, and we illustrate its implications with an application to a network of scientific citations.

Our main objective is to study how homophily patterns behave in an evolving network. Do nodes become more integrated or more segregated as they age? How does this evolution depend on the link formation process? In particular, does the network become more integrated if new connections are formed at random or if they are formed through the existing network?

To answer these questions, we study a stochastic model of network formation in which nodes come in different types and types, in turn, affect the formation of links. We accomplish this by introducing individual heterogeneity to the framework of Jackson and Rogers [19], allowing us to focus on the issue of homophily generated through specific biases in link formation. A new node is born at each time period and forms links with existing nodes. The newborn node connects to older nodes in two ways. First, she meets nodes according to a random, but potentially typebiased, process. Second, the newborn node meets neighbors of the randomly met nodes ("friends of friends"). This is referred to as the search process and can also reflect type biases. To illustrate, consider citation patterns. Typically when writing a new paper, some references are known or found by chance by the authors while others are found because they are cited in known papers. Biases arise because papers may cite references with greater frequency within their own field. We examine the long-run properties of this model and the structure of the emerging network.

The biases could arise from agents' preferences over the types of their neighbors and/or from biased meeting opportunities that agents face in connecting to each other. So, in one direction we enrich a growing network model by allowing for types and biases in connections, and in another direction we bypass explicit strategic considerations by studying a process with exogenous behavioral rules. Since in the model search goes through out-links only, strategic considerations are to some extent inherently limited, since a node cannot directly increase the probability of being found through its choice of out-neighborhood. While this may not be a good assumption in some contexts, such as business partnerships or job contacts, where search presumably goes both directions along a link, it is appropriate in other contexts, such as scientific citations where the time order of publications strictly determines the direction of search.

We wish to understand the conditions under which the network becomes increasingly "integrated" over time. We consider three different notions of integration. Under weak integration,

\footnotetext{
1 See $[15,17,21]$ for more background and discussion.
} 
nodes who are old enough are more likely to get new links than young nodes, independent of types. In this sense, age eventually overcomes bias in link probabilities. Under long-run integration, the distribution of types among the neighbors of a given node converges to the population distribution as the node ages. This is a strong property that requires biases among neighbors to eventually disappear, despite the biased formation process. Finally, under partial integration, the type distribution among neighbors moves monotonically towards the population distribution as nodes age, although it may maintain some bias in the limit. These notions capture different, but related, aspects of the idea of network integration.

Our main theoretical results are as follows. First, weak integration is satisfied whenever the probability that a given node is found increases with that node's degree. This holds in any version of our model where at least some links are formed through search and there is some possibility of connecting across types.

In contrast, long-run integration holds only when search is unbiased. That is, the random meeting process can incorporate arbitrary biases, but once the initial nodes are met, the new node chooses uniformly from the set of their neighbors, ignoring any further implication of their types. Finally, we show that under a particular condition on the biases, the process evolves monotonically and satisfies partial integration. In particular, the biases in nodes' links generally decrease with age.

To understand where this tendency towards integration comes from, consider unbiased search. Observe first that as a node ages, the proportion of its links obtained through search approaches unity, since the number of neighbors grows with age while the probability to be found at random decreases with population size. Next, note that unbiased search does not imply an absence of bias among the neighbors of randomly met nodes. Due to homophily, randomly met nodes are relatively more connected with nodes of their own types. A critical fact our analysis uncovers, however, is that bias among neighbors' neighbors tends to be lower than among direct neighbors. This is because some nodes of other types are found at random and these nodes are relatively more connected to nodes like themselves. So the set of neighbors' neighbors has a more neutral composition than the neighborhoods of same-type nodes. Network-based search increases the diversity of connections and, conversely, nodes found through search are being found by a more diverse set of nodes. And since search plays a larger role with age, older nodes are less biased in their connections.

In order to analyze network structure in more detail we consider a special, but natural, twotype specification of the model where random meetings are organized through a geographic or social space. Nodes of a given type are more likely to reside in a given location and random meetings take place without further bias in the various locations. In this model, biases in random meetings are inherently tied in a precise way to the type-distribution of the population. This feature allows us to obtain a number of further results. In particular, we derive an explicit formula relating a node's local homophily among neighbors to its age or degree. This illustrates our general results and further shows how partial and long-run integration are affected by changes in types' shares. We also study two important structural properties of the resulting network that are less tractable in the general model: degree distributions and group-level homophily. We show how to modify the existing analysis of degree distributions to account for individual heterogeneity and homophily, obtaining new insights.

In addition, we obtain results on group-level homophily consistent with empirical results presented in $[9,10]$. We find that relative group size has an important impact on how meeting biases map into aggregate properties of the network. In particular, relative homophily in the network is strongest when groups have equal size, and vanishes as the groups take increasingly unequal 
sizes. Turning to degree distributions, we find that the majority and minority groups have different patterns of interactions. In particular, for the minority group, links from their own group are on the one hand rarer due to a size effect, but on the other hand a homophilic bias pulls in the other direction, creating a tension in the overall distribution of links. However a striking result that is, in principle, testable is that the distribution of total in-links is identical for the groups independent of their relative sizes.

Moving from the theoretical analysis, we illustrate the implications of the model using data on scientific citations in journals of the American Physical Society (APS) published between 1985 and 2003. We find that the proportion of citations that a paper obtains from other papers in its own field decreases as the paper ages and becomes more cited. The observed citation patterns provide some evidence of the partial integration property and are at least partly consistent search follows a less biased (possibly unbiased) pattern in the citation process. In studying this application we are motivated by two factors. First, patterns of scientific citations have important welfare consequences as they affect the diffusion of knowledge, with impacts on different research fields. ${ }^{2}$ Previous research, such as $[13,26]$, generalizing popular concepts such as the recursive impact factor, stress that the importance of a citation relies on the paths that it allows in the network of citations. We complement this argument by considering under which conditions citations are likely to bridge scientific production across different communities. ${ }^{3}$ Second, scientific citations possess all the features of the network formation process that we study: nodes (papers) appear in chronological order and never die, they link directionally to previously born nodes, they have types (scientific classifications), and they find citations both directly and though search among the citations of other papers. ${ }^{4}$

More generally, our study contributes to a growing literature in economics and other disciplines studying the causes and consequences of homophily in social networks. Refs. [9,10] study a matching process of friendship formation. They document several empirical patterns of homophily and explain them through a combination of biases with respect to choice and chance. By design, all individuals have the same degree and age has little impact. In contrast, differences in age and degree are central to our analysis. Ref. [16] incorporates homophily into the random graph model of $[6,7] .{ }^{5}$ Again by design, homophily is not affected by degree or age in this approach. Thus, our study and these two papers study homophily patterns in networks from complementary perspectives. In particular, we provide the first study of how homophily patterns change over time and of the relation between homophily and a node's degree.

This study also advances the analysis of stochastic models of network formation. Earlier work has made great progress in explaining structural network features such as small diameter, high clustering and fat tails in degree distributions, see [1,6,7,19,23,24,37]. However, most of these studies assume homogeneous agents and neglect homophily. With respect to this literature, we

\footnotetext{
2 See, for instance, [5,14].

3 Ref. [32] studies cross-field citations in the scientific production of the 90s, for three different datasets.

4 These longitudinal aspects of citation networks have motivated the use of growing network models in previous papers including the seminal work on citation networks by Price [27,28]. Refs. [3,35], among others, find that citations on the PNAS on a 20 years interval show some aspects of a bias towards recently published papers, while Refs. [25,31], correcting for cohort size and idiosyncratic popularity, find an age effect (first mover advantage) and a frequency distribution of in-citations that are consistent with a growing network model such as the one that we develop here. Finally, Ref. [33] finds a positive correlation between homophily of out-citations and the number of in-citations, but this effect is valid only for low number of in-citations.

5 Ref. [12] uses this extension to study how homophily affects communication dynamics in networks, demonstrating explicitly one way in which homophilic structure impacts outcomes, as does [18].
} 
develop and study one of the first stochastic model of network formation incorporating individual heterogeneity.

The rest of the paper is organized as follows. Section 2 presents the model with bias only in the random meeting process. Section 3 includes the main result about long-run integration in this setting. Section 4 studies the special case of two-types and location-based bias. Section 5 studies the integration properties of the model when biases appear also in the search part of the meeting process. Section 6 contains the empirical application to citation data.

\section{Homophily in a random meeting process}

In our model, nodes are born with randomly assigned types and enter sequentially, meeting existing nodes upon entry. Meetings result in (directed) links. Meetings take place through two distinct processes, which we refer to as random and search. The meeting processes depend on the types of the nodes involved. In this section, we study the impact of type-based biases on the random meeting process.

\subsection{The model}

Time is indexed by $t=1,2, \ldots$ In each period a new node is born. We index nodes by their birth dates, so that node $t$ is born in period $t$.

Nodes have "types", with a generic type denoted $\theta$ belonging to a finite set $\Theta$ (with cardinality $H$ ). A newborn's type is randomly drawn according to the time invariant probability distribution $p$ (so that types are i.i.d., across time).

A newborn node sends $n>1$ (directed) links to the nodes that were born in previous periods. Of these $n$ links, a fraction $m_{r}$ selects nodes according to a type-dependent random process ${ }^{6}$; these nodes are called "parents". The remaining fraction $m_{s}=1-m_{r}$ selects nodes among the neighbors of the $n m_{r}$ parents that have been found via the random process; we refer to this second part of the process as "search". ${ }^{7}$ We define $\sigma \equiv m_{r} / m_{s}$ to be the ratio of the number of links formed by the random process to the number of links formed by the search process.

Looking first at the random part of the process, we denote by $p\left(\theta, \theta^{\prime}\right)$ the probability that a link sent by a node of type $\theta$ reaches a node of type $\theta^{\prime}$. Among nodes of type $\theta^{\prime}$, the link is formed uniformly at random, so there is no further discrimination in this part of the process. If the random meeting process were unbiased, the probability $p\left(\theta, \theta^{\prime}\right)$ would equal the share $p\left(\theta^{\prime}\right)$ of $\theta^{\prime}$ agents in the system. When $p\left(\theta, \theta^{\prime}\right) \neq p\left(\theta^{\prime}\right)$ we say that there is bias. This can be interpreted in different ways. One can view the bias as a reduced form for preferences that nodes have over the type of connections they form. The case of "homophilistic" preferences for type $\theta$ is then captured by a situation in which $p(\theta, \theta)>p(\theta)$. The bias could also arise from constraints in the meeting process, or from spatial differentiation, as in the location model that we will analyze in Section $4 .{ }^{8}$

Turning now to the search part of the process, the way in which friends are drawn from parents' neighborhoods may be, in principle, either biased or unbiased. Much of the paper will study

\footnotetext{
$6 m_{r} n$ is an integer in the underlying process, but allowed to be arbitrary in the mean-field continuous-time approximation we analyze.

7 In the underlying process, if some node is found to which the newborn is already connected, then the node is redrawn. If there are too few new nodes in the neighborhoods of the nodes found in the first part of the process, then the random nodes are redrawn. To ensure that the process is well defined, we begin with a set of $n^{2}$ nodes in a sequence, each connected to all predecessors.

8 See [9-11] for more details on other models that can justify this reduced form.
} 
a process with biases only in the random part, so that in the search part, links are formed according to a uniform distribution on the set of parents' neighbors. This assumption has natural interpretations and various applications. It applies, for instance, to cases where agents face a bias in meeting strangers, but then get to meet the "friends" of their new friends without bias. When the original bias in meetings comes from biased opportunities, this seems to be a natural assumption; when the bias originates in preferences, it may still be the case that this bias tends to vanish when meetings are mediated by friends. In Section 4 we will explicitly analyze a model that relates these biases to location-based differences in the meeting process. When search is itself biased, so that the additional $\mathrm{nm}_{\mathrm{s}}$ nodes are found among parents' neighbors using a type-dependent probability distribution, two types of biases are naturally defined: a bias that discriminates according to the types of the parents through which search is made, and a bias that discriminates according to the types of the parents' neighbors. Which type of bias is more appropriate depends on the instance of network one has in mind, and leads to formally different models of link formation. In Section 5 we study the case of biased search and its consequences for integration.

Before formally deriving the dynamics of the various processes, in the next section we propose three notions of integration that measure the extent to which the bias in the random and/or search process translates into biases in the long run type-patterns of link formation.

\subsection{Integration}

The definitions we provide capture different aspects of integration, focusing on how a node's type-pattern of connections evolves with age, and whether it gets progressively more (or less) integrated with the rest of the network.

It is important to note that there are two different aspects of integration: the evolution of newborns' newly formed links (out-links) and the evolution of older nodes' incoming links. These will exhibit different dynamics. Given the bias in the random part of the network formation process, it is clear that there will always be some bias in the out-links of newborn nodes. The main questions with regard to the out-links thus pertain to how the links formed by search behave over time, and this is related to the question of how the in-links of older nodes behave.

All three notions of integration discussed here pertain to the behavior of in-degrees of nodes. Out-degree dynamics are studied in Sections 3.3 and 4.3.

Our first notion requires, in particular, that old enough nodes are found by newborn nodes with higher probability than younger nodes, independently of the types of the nodes involved.

Definition 1. The network formation process satisfies the weak integration property if for every $t_{0}$, there exists $t>t_{0}$ such that, for all $t^{\prime} \geqslant t$ and for all $\theta \in \Theta$, the node born at time $t^{\prime}$ has a lower probability than node $t_{0}$ to receive a link from a node of type $\theta$ born at time $t^{\prime}+1$.

Note that this form of integration requires that an old enough node of type $\theta$ ends up receiving a link from a newborn node of type $\theta^{\prime}$ with a higher probability than a young enough node of the same type $\theta^{\prime}$ as the newborn. So, even if link formation probabilities are biased in favor of similar nodes (homophily), old enough nodes are found more often even when of a different type than the newborn.

This form of integration is rather weak, and does not bear implications on the typecomposition of any given node's in-degree. Our second notion of integration requires that as nodes age, their local neighborhood grows to represent more and more the type-composition of the population. It is therefore a "monotonicity" property, requiring that integration, here defined 
in terms of how close the composition of neighbors' types is to what would obtain in the unbiased case, grows with age.

Definition 2. The network formation process satisfies the partial integration property if for every node $t_{0}$ the fraction of each type $\theta$ in the in-degree of $t_{0}$ is weakly closer to $\theta$ 's population share at time $t^{\prime}$ than at time $t$, for $t^{\prime}>t$, and strictly closer for some types.

So, under partial integration, the in-neighbors of an agent become more and more representative of the overall population as time elapses.

Our final notion of integration is stronger ${ }^{9}$ and requires that nodes eventually attract in-links according to population shares.

Definition 3. The network formation process satisfies the long-run integration property if for every node $t_{0}$ the proportion of each type $\theta$ in the in-degree of $t_{0}$ converges to $\theta$ 's population share as node $t_{0}$ ages.

In other words, in the long run any surviving difference in the proportion of links received by old nodes from different types is due only to the distribution of types in the population, and the biases in link formation have no consequences for eventual in-degree patterns.

\section{Integration with biased random meetings and unbiased search}

\subsection{Model dynamics}

A benchmark model to study long-run integration properties of link formation is one where only the random part of the process is biased, and no further bias is present in the search part of the process. More precisely, the search process is unbiased in the sense that additional ties are found through a uniform sample among parents' neighbors, but remains indirectly biased through the bias that the random process has induced on the type-composition of the parents' neighborhoods. This model allows for a clear understanding of the mechanics that lead to integration, and why and when integration may fail.

We study a continuous-time approximation of the model, using the techniques of mean-field theory. This provides approximations and limiting expressions of the process that ignore starting conditions and other short-term fluctuations that can be important in shaping finite versions of the model, and so the results must be viewed with the standard cautions that accompany such approximations and limit analysis. We consider the expected change in the discrete stochastic process as the deterministic differential of a continuous-time process.

Let us first look at the probability that node $j$ is found by newborn node $t+1$. This depends on the shape of the network that has formed up to time $t$. In particular, it depends on the type-profile of in-neighbors of $j$ at time $t$, and on the bias of the newborn node towards such types. Since search is not type-biased, each link that agent $t+1$ forms through search is drawn from a uniform distribution over the set of all neighbors of all parent nodes that agent $t+1$ has found at random.

Letting $P_{j}^{t}\left(\theta_{t}, \theta_{j}\right)$ denote the probability that a node born in period $j$ of type $\theta_{j}$ receives a link from a node of type $\theta_{t}$ born at time $t>j$, the following expression is a mean-field approximation

\footnotetext{
9 By stronger we do not mean that it is a necessary condition for the partial integration property defined above. One could think of partial integration as a criterion of monotonicity of a function in one variable, while the long-run integration defined below is a criterion of convergence, that could however also be non-monotonic.
} 
of the overall linking probability:

$$
P_{j}^{t+1}\left(\theta, \theta_{j}\right)=n m_{r} \frac{p(\theta) p\left(\theta, \theta_{j}\right)}{t p\left(\theta_{j}\right)}+n m_{s} \sum_{\theta^{\prime} \in \Theta} p(\theta) p\left(\theta, \theta^{\prime}\right) \frac{\sum_{\lambda=j}^{t} P_{j}^{\lambda}\left(\theta^{\prime}, \theta_{j}\right)}{t p\left(\theta^{\prime}\right)} \frac{1}{n} .
$$

The first term on the right-hand side captures the probability of node $j$ being found at random. The probability that node $t+1$ is of type $\theta$ and links at random to a node of type $\theta_{j}$ is $p(\theta) p\left(\theta, \theta_{j}\right)$. This is divided by the number of nodes of type $\theta_{j}$ at time $t$ which, under a meanfield approximation, is equal to $\operatorname{tp}\left(\theta_{j}\right)$. It is then multiplied by the number of links formed at random, $n m_{r}$.

The second term is the probability of node $j$ being found through search. It is given by the number of search links $\left(n m_{s}\right)$ formed by the node born at $t+1$, times the sum, over all possible types $\theta^{\prime}$, of the probabilities that $j$ is found through a node of type $\theta^{\prime}$. For each possible type $\theta^{\prime}$, this probability is given by the joint probability of the following events (corresponding to the four terms in the first summation over types): (i) the newborn node is of type $\theta$; (ii) it forms a link with a $\theta^{\prime}$-type node; (iii) the $\theta^{\prime}$-type node has linked to $j$ since $j$ was born ${ }^{10}$; (iv) among the $n$ neighbors of this $\theta^{\prime}$-type node, that exactly $j$ is found.

It is useful to express the terms of the above formula in a compact way. For all $\theta, \theta^{\prime}$ we write

$$
B_{r}\left(\theta, \theta^{\prime}\right) \equiv p(\theta) \frac{p\left(\theta, \theta^{\prime}\right)}{p\left(\theta^{\prime}\right)} .
$$

Note that the ratio $\frac{p\left(\theta, \theta^{\prime}\right)}{p\left(\theta^{\prime}\right)}$ in the above expression is a measure of the bias that type $\theta$ applies to type $\theta^{\prime}$, so that when this ratio is 1 there is no bias, while when it is greater (less) than 1 there is a positive (negative) bias of type $\theta$ towards type $\theta^{\prime}$. In the case of no bias, $B_{r}\left(\theta, \theta^{\prime}\right)$ is simply the probability of birth of a type $\theta$ node, and $P_{j}^{t+1}\left(\theta, \theta_{j}\right)$ is $n$ times the joint probability that the newborn node is of type $\theta$ and that node $j$ is found by drawing uniformly at random from a population of $t$ nodes. We can decompose the matrix $\mathbf{B}_{r}$ as the product of two matrices $\mathbf{A}$ and $\mathbf{Q}$, where $\mathbf{A}$ may be seen as a transition matrix of a Markov process (a Markov matrix), ${ }^{11}$ and $\mathbf{Q}$ is a diagonal matrix where the diagonal is a probability vector:

$$
\mathbf{B}_{r}=\mathbf{Q} \mathbf{A} \mathbf{Q}^{-1}
$$

with

$$
\mathbf{A}_{\theta \theta^{\prime}}=p\left(\theta, \theta^{\prime}\right) \text { and } \mathbf{Q}=\left(\begin{array}{ccc}
p(1) & \cdots & 0 \\
\cdots & \cdots & \cdots \\
0 & \cdots & p(H)
\end{array}\right) .
$$

Using the matrix $\mathbf{B}_{r}$, Eq. (1) becomes

$$
\mathbf{P}_{t_{0}}^{t+1}=\frac{n m_{r}}{t} \mathbf{B}_{r}+\frac{m_{s}}{t} \mathbf{B}_{r} \sum_{\lambda=t_{0}}^{t} \mathbf{P}_{t_{0}}^{\lambda}
$$

where $\sum_{\lambda=t_{0}}^{t} \mathbf{P}_{t_{0}}^{\lambda}$ expresses the expected in-degree, type-by-type, after time $t$ for a node born at time $t_{0}$.

\footnotetext{
$\overline{10}$ Note that this ratio has the total (expected) number of links received by agent $j$ from $\theta^{\prime}$ agents up to time $t$ as numerator, and the total number of $\theta^{\prime}$ nodes in the system at time $t$ as denominator.

11 In Appendix A we derive some general results on Markov matrices that will be useful in Appendix B, where we prove our propositions.
} 
We define

$$
\Pi_{t_{0}}^{t} \equiv \sum_{\lambda=t_{0}}^{t} \mathbf{P}_{t_{0}}^{\lambda}
$$

With a continuous approximation:

$$
\frac{\partial}{\partial t} \Pi_{t_{0}}^{t}=\mathbf{P}_{t_{0}}^{t+1}
$$

We study Eq. (3) in terms of ordinary differential equations in matrix form:

$$
\frac{\partial}{\partial t} \Pi_{t_{0}}^{t}=\frac{n m_{r}}{t} \mathbf{B}_{r}+\frac{m_{s}}{t} \mathbf{B}_{r} \Pi_{t_{0}}^{t}
$$

with the initial condition

$$
\Pi_{t_{0}}^{t_{0}}=\mathbf{0}
$$

From now on we will always assume that $\mathbf{B}_{r}$ is invertible (so that the specification of types is not redundant). With this assumption, the unique solutions to these differential equations are the following:

$$
\Pi_{t_{0}}^{t}=n \frac{m_{r}}{m_{s}}\left(\left(\frac{t}{t_{0}}\right)^{m_{s} \mathbf{B}_{r}}-\mathbf{I}\right),
$$

where a constant to the power of a matrix is defined as follows:

$$
\left(\frac{t}{t_{0}}\right)^{m_{s} \mathbf{B}_{r}}=e^{\left(m_{s} \log \left(\frac{t}{t_{0}}\right) \mathbf{B}_{r}\right)}=\sum_{\mu=0}^{\infty} \frac{\left(m_{s} \log \left(\frac{t}{t_{0}}\right) \mathbf{B}_{r}\right)^{\mu}}{\mu !} .
$$

\subsection{Integration}

Let us test the various notions of integration on this model with unbiased search.

It is clear that the model with $m_{r}=1$ cannot satisfy weak integration. We show instead that whenever there is some degree of search $\left(m_{r}<1\right)$ weak integration is satisfied. In fact, in Section 5 we strengthen this result to show that weak integration is still satisfied when search is biased as well.

Proposition 1. If $m_{r}<1$, the model with unbiased search satisfies the weak integration property.

The proof (which appears, along with the proofs of our other results, in Appendix B) shows that the weak integration property is not specific to the unbiased search model. Indeed, various models in which the in-degree of a node determines the probability of being found by a newborn node in a sufficiently increasing manner would give the same result. Moreover, search is not needed for this type of dependence to take place. Another model with "type-biased" preferential attachment in which the probability of receiving a link is positively correlated with a node's indegree, and which exhibits the same weak integration property, is discussed in the conclusion of [11].

Partial and long-run integration are, again, not satisfied when $m_{r}=1$. The next propositions show that, otherwise, the long-run integration property is always satisfied by the model, while the partial integration property needs an additional assumption. 
Proposition 2. If $m_{r}<1$, the model with unbiased search satisfies the long-run integration property.

Partial integration, instead, occurs under an additional condition. Consider a Markov matrix M. As formally stated in Appendix A, writing $\overline{\mathbf{M}} \equiv \lim _{\mu \rightarrow \infty} \mathbf{M}^{\mu}$, we say that $\mathbf{M}$ satisfies the monotone convergence property if, for every pair $i, j \in\{1, \ldots, H\}$, and for every $\mu \in \mathbb{N}$, the element $M_{i j}^{\mu}$ satisfies:

$$
\begin{aligned}
& \text { 1. if } M_{i j}>\bar{M}_{i j} \text {, then } M_{i j} \geqslant M_{i j}^{\mu} \geqslant M_{i j}^{\mu+1} \geqslant \bar{M}_{i j} \text {; } \\
& \text { 2. if } M_{i j}<\bar{M}_{i j} \text {, then } M_{i j} \leqslant M_{i j}^{\mu} \leqslant M_{i j}^{\mu+1} \leqslant \bar{M}_{i j} \text {. }
\end{aligned}
$$

The monotone convergence property captures the idea that transition probabilities are monotone over time. Even with a strictly positive transition matrix, this condition does impose additional restrictions. ${ }^{12}$ It is beyond the scope of this paper to find general or even necessary conditions for monotone convergence of Markov matrices.

We then have the following result.

Proposition 3. If $m_{r}<1$ and $\mathbf{A}$ satisfies the monotone convergence property, then the model with unbiased search satisfies the partial integration property.

Let us focus on the intuition behind the long-run integration property of the model with unbiased search. To fix ideas, let us examine the case in which random probabilities have a homophilous bias. A given node can be found by a newborn node of a different type via search in different ways: one is that the newborn finds a neighbor of the given node that is of the same type as the newborn, and another is that the newborn finds a neighbor of the given node that is of the same type as the given node. The first way is relatively more likely given the homophilous bias in the random part of link formation, but the fact that this can also occur via the second route leads this process to be less biased over time. Once the process has become less biased, it even easier to be found by nodes of other types, and so the neighborhood becomes even less biased, and this trend reinforces itself leading to an unbiased process in the limit. To summarize, as a node ages it becomes more of a "hub", attracting many links from all types in the search process. This property, that also underlies the weak integration property, together with unbiased search further decreases the bias in the in-degree of hubs. As a result, the type-composition of new connections becomes even less biased for these hubs, and eventually the bias is eliminated.

The way in which an individual's neighborhood composition limits to the population frequencies as it ages is non-trivial. Notice that if a particular individual became connected to a large proportion of others over time, then his neighborhood would necessarily approximate population frequencies. However, we emphasize that in our model, even though an individual's degree grows without bound, the proportion of others to whom he is connected still vanishes over time, so this

\footnotetext{
12 As a simple illustrating example, consider a Markov process with three states where transitions from state 1 to state 2 , 2 to 3 , and 3 to 1 occur with high probability, and with the other transitions occurring with small but positive probabilities. Then in one period going from 1 to 2 is likely, but then it is unlikely to occur in two periods or three periods, but more likely in four periods, and so forth. Things eventually converge to equal likelihood on all states, but convergence is not monotone. One can also find such examples that are more complicated where homophily is present.
} 
effect is not what drives integration. This happens because the entry rate of new individuals is constant, while the probability for existing individuals to acquire a new link in any given period goes to zero.

Finally, we remark that, while the neighborhood of every node approaches a composition that reflects the aggregate population frequencies, it converges to that distribution from a point that is affected by biases in the link formation process. Since those links are perpetually being formed and are subject to biases, the system never approaches a network that has unbiased link patterns. Rather, it is always the oldest nodes in the system that have the least biased neighborhoods. In fact, one way to see the persistent bias is to focus on out-degree rather than in-degree. Thus, we turn now to analyzing links by tracking where they originate.

\subsection{On the dynamics of out-degrees}

So far we have mostly focused on the dynamics of agents' in-degree. Of course, out- and in-degree dynamics are intimately related, as the search part of young nodes' out-degree will consist predominantly of old nodes, with respect to whom the search part of the process is both directly and indirectly unbiased (see Section 4.3). Here we take a close look at the composition of out-degrees and how they evolve over time. This is of interest not only to better understand integration, but also to shed light on the evolution of homophily, that is, the tendency to form ties with agents of the same type.

We first look at the steady state composition of the out-degree. Let us denote by $d_{i j, t}$ the proportion of links that originate from a node of type $i$ born at time $t$ that are directed towards nodes of type $j$. The evolution of these proportions is given by

$$
d_{i j, t+1}=\left(1-m_{s}\right) B_{r}(i, j)+m_{s} \sum_{h=1}^{H} B_{r}(i, h) \frac{\sum_{\tau=1}^{t} d_{h j, \tau}}{t} .
$$

The out-degree depends on the random part (first term) and on the search part (second term) through the average out-degree of existing nodes. In matrix form, this is written as follows:

$$
\mathbf{D}_{t+1}=\left(1-m_{s}\right) \mathbf{B}_{r}+m_{s} \mathbf{B}_{r} \frac{\sum_{\tau=1}^{t} \mathbf{D}_{t}}{t} .
$$

To get a feeling for the limit of this process, it is useful to examine the steady state $\overline{\mathbf{D}}$ of this system. The steady-state is such that the out-degree of each type remains unchanged in time:

$$
\overline{\mathbf{D}}=\left(1-m_{s}\right) \mathbf{B}_{r}+m_{s} \mathbf{B}_{r} \overline{\mathbf{D}} .
$$

Proposition 4. If $m_{s}<1$, then the steady state Eq. (9) has a unique solution $\overline{\mathbf{D}}$, and the system in (8) converges to $\overline{\mathbf{D}}$.

For $m_{s}<1$, the second term approaches the null matrix as $t \rightarrow \infty$. As long as the matrix $\mathbf{D}_{1}$ is more biased than the steady state $\overline{\mathbf{D}}$ (which is true for $\mathbf{D}_{1}=\mathbf{A}$ ), the bias in excess of the steady state decreases with time, vanishing in the long run (see also Appendix A).

This means that the biases in the out-links formed by agents decrease over time, consistent with the homogenization of the search process and the in-degree of older nodes which are dominating the search part of the process. However, unlike the case of the in-degree of old nodes, full homogenization does not occur even in the limit, since the random part of the out-degree formation does not vanish over time. 


\section{Location-based biases}

In this section we consider a specific form of bias in random meetings, and restrict the analysis to two types for simplicity. By making explicit how the bias in random meetings is generated, we accomplish two goals. First, we generate a closed-form expression that describes the integration of individuals as they age. This formula allows us to study in more detail the integration process, and provides parameters which can be empirically estimated. Second, we obtain additional results on other features of the network, specifically on aggregate homophily at the group level and in-degree distributions that are type-sensitive. For each of these categories of results, the location-based nature of the meeting biases allows us to study the impact of changes in population frequencies on the structure and properties of the emerging networks.

Nodes belong to one of two types: $\theta_{1}$ and $\theta_{2}$. With an abuse of notation, let $p\left(\theta_{1}\right)=p$ and $p\left(\theta_{2}\right)=1-p$ in this section. There are two locations $L^{1}$ and $L^{2}$. All biases in the meeting process are captured by the parameter $\gamma \in[1 / 2,1]$, which represents the probability that a $\theta_{i}$ node goes to location $L^{i}, i=1,2 .{ }^{13}$ Once assigned to a location, each agent meets $m_{r} n$ nodes uniformly at random among all individuals present at this location. Thus, it is simply the resulting composition of types in the two locations that permits any type-dependent biases in the model. We maintain the assumption that the search part of the process is unbiased.

At any time $t$, the expected number of $\theta_{1}$ nodes at $L^{1}$ is $p \gamma t$, while the expected number of $\theta_{2}$ nodes in $L^{1}$ is $(1-p)(1-\gamma) t$. Thus, the proportion of $\theta_{1}$ nodes in $L^{1}$ is $\frac{p \gamma}{p \gamma+(1-p)(1-\gamma)}$ while the proportion of $\theta_{1}$ nodes in $L^{2}$ is $\frac{p(1-\gamma)}{p(1-\gamma)+(1-p) \gamma}$. The probability that a node of type $\theta_{1}$ links at random to a node of the same type is thus:

$$
p\left(\theta_{1}, \theta_{1}\right)=\gamma \frac{p \gamma}{p \gamma+(1-p)(1-\gamma)}+(1-\gamma) \frac{p(1-\gamma)}{p(1-\gamma)+(1-p) \gamma}
$$

and $p\left(\theta_{2}, \theta_{2}\right)$ is obtained by symmetry exchanging $p$ and $1-p$.

Thus, the model generates a simple explicit relation between population frequencies and random meeting biases, controlled by the parameter $\gamma$. Note that when $\gamma=1 / 2$, locations are independent of types and there is no bias: $p\left(\theta_{i}, \theta_{i}\right)=p\left(\theta_{i}\right)$. In contrast, when $\gamma>\frac{1}{2}$ random meetings are biased towards own group and $p\left(\theta_{i}, \theta_{i}\right)>p\left(\theta_{i}\right)$. At the extreme when $\gamma=1$, locations are perfectly correlated with types and individuals meet others only from their own group so that $p\left(\theta_{i}, \theta_{i}\right)=1$. This allows us to derive a number of comparative statics results with respect to population frequencies below.

\subsection{Explicit formulas for long-run integration}

Using the expressions above we have

$$
B_{r}=\left(\begin{array}{cc}
p\left(\theta_{1}, \theta_{1}\right) & \frac{p}{1-p}\left(1-p\left(\theta_{1}, \theta_{1}\right)\right) \\
\frac{1-p}{p}\left(1-p\left(\theta_{2}, \theta_{2}\right)\right) & p\left(\theta_{2}, \theta_{2}\right)
\end{array}\right) .
$$

We note that Proposition 3 (together with Lemma 5 in Appendix A) implies that if $m_{r}<1$, then the location-based model always satisfies the partial integration property, because $p\left(\theta_{1}, \theta_{1}\right) \geqslant \frac{1}{2}$ and $p\left(\theta_{2}, \theta_{2}\right) \geqslant \frac{1}{2}$.

13 The analysis below assumes away some implicit correlations in the meeting process described here. This is as if (modulo matching issues) each new node in $g^{i}$ spends a proportion $\gamma$ of his time in $L^{i}$, and the probability of meeting any existing node is proportional to the time spent with it in the same location. 
We can now solve Eq. (4) in terms of $p$ and $\gamma$ to obtain

Lemma 1. The in-link composition at time $t$ of a type $\theta_{1}$ node born at time $t_{0}$ is

$$
\begin{aligned}
& \Pi_{t_{0}}^{t}(1,1)=n \frac{m_{r}}{m_{s}}\left(p\left(\frac{t}{t_{0}}\right)^{m_{s}}+(1-p)\left(\frac{t}{t_{0}}\right)^{b m_{s}}-1\right), \\
& \Pi_{t_{0}}^{t}(2,1)=n \frac{m_{r}}{m_{s}}(1-p)\left(\left(\frac{t}{t_{0}}\right)^{m_{s}}-\left(\frac{t}{t_{0}}\right)^{b m_{s}}\right),
\end{aligned}
$$

with the analogous expressions for type $\theta_{2}$, where

$$
b \equiv p\left(\theta_{1}, \theta_{1}\right)+p\left(\theta_{2}, \theta_{2}\right)-1=1-\frac{\gamma(1-\gamma)}{p(1-p)(2 \gamma-1)^{2}+\gamma(1-\gamma)} .
$$

We can now show that the location model generates a simple, explicit relationship between integration and in-degree at the individual level. This allows us to illustrate the results from the previous section and to obtain further predictions on the shape of integration. In particular, we find that the amplitude of integration tends to be lower in larger groups.

We denote an individual's in-degree by $k$, which is a function of the node's entry date $t_{0}$ and the individual's age. Then $r^{j}(k)$ denotes the individual share of same type in-links for a node of type $\theta_{j}$ at the time when its degree is $k$.

Proposition 5. Suppose that biases are location-driven. Then, $r^{j}(k)$ is described by

$$
r^{j}(k)=p\left(\theta_{j}\right)+\left(1-p\left(\theta_{j}\right)\right) \frac{\left(1+k m_{s} /\left(n m_{r}\right)\right)^{b}-1}{k m_{s} /\left(n m_{r}\right)} .
$$

The individual share of same type in-links is thus decreasing with $k$ and convex in $k$, converging asymptotically to the population shares. Moreover, for $k>0, \partial r^{j} / \partial p^{j}(k)>0$ and $\partial^{2} r^{j} / \partial p^{j} \partial k(k)>0$.

Thus, consistent with the general long-run integration result of Proposition $2, r^{j}(k)$ converges to the population frequency $p\left(\theta_{j}\right)$ as $k$, and hence time, diverge. The convergence is monotonic, and so satisfies the property of partial integration described in Proposition 3. Notice that by application of Lemma 5 in Appendix A, one could demonstrate partial integration without the explicit solution contained in Proposition 5. However, the formula delivered by Proposition 5 allows us to derive some further implications. First, the relation between $r^{j}(k)$ and in-degree is convex, so its decrease with age takes place at a decreasing rate. Second, and perhaps more importantly, the relation between $r^{j}(k)$ and degree tends to be flatter in larger groups; the difference in integration between low-degree and high-degree nodes is smaller in larger groups. Third, this function could be readily fitted to data. In contexts where information on how links are formed is lacking, this could provide the basis of an empirical analysis of the model. This approach is illustrated in [4].

\subsection{Cumulative link distributions}

We turn now to a more detailed discussion of the distribution of links across nodes in the network. Proposition 5 makes explicit the relationship between the degree of an individual and the local composition of its in-neighbors, demonstrating, in particular, the properties of partial 
and long-run integration. Integrating that relationship in order to obtain a measure of grouplevel homophily requires knowing the distribution of in-degree across individuals. This section is concerned precisely with analyzing those degree distributions, which have become a cornerstone of social network analysis.

Even with only two groups, capturing the distribution of links becomes substantially more complex, relative to the one-group case, as nodes can connect to both same- and different-type nodes, and one wants to keep track of the different kinds and sources of links. In this context, we can keep track of seven different degree distributions rather than one. Define $F_{i j}$ as the distribution of the in-degrees of type $\theta_{i}$ nodes paying attention only to links coming from nodes of type $\theta_{j}, i, j=1,2$. Then $F_{1}$ and $F_{2}$ are the standard in-degree distributions of $\theta_{1}$ and $\theta_{2}$ nodes (ignoring the types of neighbors), and finally $F$ is the total in-degree distribution of the entire society.

We observe that as a consequence of Lemma 1, all of the degree distributions have a power-law upper tail, as has been documented extensively in empirical contexts, starting from [1]. Further, we are able to make predictions about how the distribution of links from own- and other-group nodes relate to each other, making clear the importance of whether a node is in the majority or minority group. Finally, we show that changing the bias in location-based meetings causes the distributions to shift in the sense of first order stochastic dominance (Proposition 7).

To begin the analysis consider, for example, $F_{1}$. Observe that $F_{1}(k)=1-t_{0} / t$, where $t$ is an arbitrary time period and, by definition, $t_{0}$ is the node that has in-degree $k$ at time $t$. $t_{0}$ can be solved for under the mean-field approximation. This defines $F_{1}$ implicitly as a function of $k$, and an analogous method works for the other distributions. While these equations do not usually yield closed-form solutions, they still allow us to derive important properties of the degree distributions. Our first such result orders the degree distributions as the number of out-links is varied.

We first ask how $F_{i 1}$ and $F_{i 2}$ compare to each other. That is, we focus on one group $\theta_{i}$, and for those nodes we compare the (distributions of) the number of links coming from the own group and the other group. We find that the relationship depends on the size of group $i$.

Proposition 6. Fix $p>1 / 2$ so that the majority group is group 1 . Then:

(i) $F_{11} F O S D F_{12}$;

(ii) If $\gamma<1$, then $F_{22}$ never FOSD $F_{21}$;

(iii) $F_{21} F O S D F_{22}$ if and only if $b \leqslant \frac{2 p-1}{2 p}$;

(iv) $F_{1}=F_{2}$.

These results express the interplay of two effects. On the one hand, there is a direct size effect through which nodes receive more links from the larger group. On the other hand, homophily leads nodes to receive relatively more links from nodes of the same group. In the larger group, both effects are aligned which implies that $F_{11}$ FOSD $F_{12}$. In the smaller group, however, these effects pull in opposite directions.

The third item in the proposition says that if homophily is not too large, the size effect dominates and $F_{21}$ FOSD $F_{22}$. The condition in part (iii) requires that $b$, and hence $\gamma$, be lower than or equal to some threshold value. ${ }^{14}$ We can see that this threshold is increasing in $p$. As the size

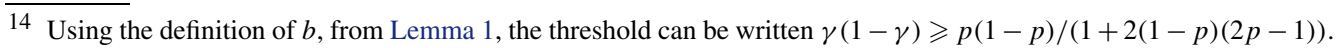


of the larger group increases, the size effect becomes relatively more important and $F_{21}$ ends up dominating $F_{22}$ for a larger range of the parameters. In contrast, the second item says that even if homophily is large, as long as it is not perfect $(\gamma<1)$, the homophily effect cannot dominate the size effect. The explanation lies with nodes of high degree. We can show that, in the upper tail, $F_{22}$ always lies above $F_{21}$. In other words, the size effect dominates for the hubs of the smaller group, and they tend to get relatively more connections from nodes of the larger group. This is related to partial integration: the largest degree nodes are more integrated with respect to their in-degree. In other words, the hubs in the minority group have the greatest proportion of their in-neighbors from the majority group.

The last part provides a particular empirical prediction, as independent of the homophily biases, the relative group sizes, and the proportion of links formed through the random meeting process, the in-degree distributions of the two groups must be identical.

The final result in this section describes how the distributions of inter- and intra-group links respond to changes in the meeting bias.

Proposition 7. Assume biases are location-driven. Fix $p, m$ and $\sigma$ and take $\gamma<\gamma^{\prime}$. Let $F_{i j}$ be the distributions corresponding to $\gamma$ and let $F_{i j}^{\prime}$ be the distributions corresponding to $\gamma^{\prime}$, for $i, j=1$, 2. Then $F_{11}^{\prime}$ and $F_{22}^{\prime}$ strictly FOSD $F_{11}$ and $F_{22}$, while $F_{21}$ and $F_{12}$ strictly FOSD $F_{21}^{\prime}$ and $F_{12}^{\prime}$.

When the meeting bias increases, no matter the group sizes, individuals tend to form more links within their own groups, and fewer links across groups.

\subsection{Long run homophily and group size}

This section complements the general analysis of Section 3.3 with a detailed inspection of the steady state out-degree composition in the two-type location-based model. In this specific context, our aim is to understand how the built-in homophily in random meetings translates into biased long run proportions of out-links that stay within a group, and how these proportions relate to groups' frequencies. Proposition 8 below tells us that group-level homophily is strongest when the groups have nearly equal sizes, and vanishes at the extreme when one group dominates society. Further, Corollary 1 tells us that, for given population frequencies, group-level homophily is stronger when the bias in random meetings or the relative prevalence of random meetings is higher.

In preparation for this result, define the homophily index $H\left(\theta_{i}\right)$ as the expected proportion of the links formed by a new $\theta_{i}$ node that are with same-type nodes. At the steady state, $H\left(\theta_{1}\right)$ and $H\left(\theta_{2}\right)$ satisfy the following equation

$$
H\left(\theta_{1}\right) n=m_{r} n p\left(\theta_{1}, \theta_{1}\right)+m_{s} n\left[H\left(\theta_{1}\right) p\left(\theta_{1}, \theta_{1}\right)+\left(1-H\left(\theta_{2}\right)\right)\left(1-p\left(\theta_{2}, \theta_{2}\right)\right)\right],
$$

as well as its symmetric counterpart. We know from the results of Section 3.3 that the steady state solution will be greater than $p\left(\theta_{1}\right)$, as the bias in out-links does not vanish with time, since the random meeting process always constitutes a non-trivial portion of out-degree. This equation decomposes the expected number of links formed within group as the sum of two terms capturing links formed at random and links formed through search. At random, this number is by definition proportional to the probability $p\left(\theta_{1}, \theta_{1}\right)$. Through search, there are two ways to connect within the group, depending on whether the intermediary node is of the same type or not. Solving these equations yields, for type $\theta_{1}$, 


$$
H\left(\theta_{1}\right)=\frac{p\left(\theta_{1}, \theta_{1}\right) \sigma+1-p\left(\theta_{2}, \theta_{2}\right)}{\sigma+2-p\left(\theta_{1}, \theta_{1}\right)-p\left(\theta_{2}, \theta_{2}\right)},
$$

recalling that $\sigma=m_{r} / m_{s}$ represents the ratio of the number of links formed at random to the number of links formed through search. Combining this expression with Eq. (10), we obtain an explicit formula linking group homophily and population frequencies, controlled by the ratio of random to search meetings $\sigma$ and the bias parameter $\gamma$. In particular, we can see that $H\left(\theta_{i}\right)$ is increasing in $\sigma$. As $\sigma$ tends to zero and search dominates the network's formation, $H\left(\theta_{i}\right)$ tends to $p\left(\theta_{i}\right)$, while when $\sigma$ becomes large and random meetings dominate, $H\left(\theta_{i}\right)$ tends to $p\left(\theta_{i}, \theta_{i}\right)$.

This means that the larger the role of search in the network formation process, the more integrated the society becomes, consistent with the intuition obtained from the general setting. This provides an expression at the group-level of the idea that search tends to reduce the imposed bias in meetings. The reason for this is that nodes found through search are more likely to be of the other type than nodes found at random, due to the possibility that the intermediary node is of the other type. As search dominates, homophily disappears completely and links are formed according to population frequencies. On the other hand, when the random meeting process dominates, links are formed according to the probabilities determined by the location-based meetings.

To analyze how group-level homophily varies with group size, we find it useful to look at a normalized index introduced by [8] (see [9]). Define relative homophily (or imbreeding homophily) as follows

$$
I H\left(\theta_{i}\right)=\frac{H\left(\theta_{i}\right)-p\left(\theta_{i}\right)}{1-p\left(\theta_{i}\right)} .
$$

Relative homophily is positive when a group forms a higher proportion of its links within the group than would be implied by the population sizes, and is normalized to have a maximal value at unity. Again, from the results of Section 3.3, relative homophily will be positive in steady state. However, we can now demonstrate a more detailed relationship between relative homophily and relative group size. The following result shows how relative homophily changes as the composition of society varies.

Proposition 8. IH 1 is symmetric around $p=1 / 2$. It is equal to zero at $p=0$ and 1 ; it increases from $p=0$ to $p=1 / 2$, and decreases from $p=1 / 2$ to $p=1$, and is concave.

Thus, in the extreme cases where one group dominates society, relative homophily disappears. Natural mixing occurring at each location tends to homogenize meetings, and this effect overcomes the impact of location biases when sizes are asymmetric. In all other cases, however, relative homophily is positive, and is strongest for intermediate size groups, reaching a maximum when the groups have equal size. Interestingly, the equilibrium mixing model of [9] generates an analogous result through a different analysis, and this prediction is supported empirically looking at racial composition in the AddHealth data.

The next result shows how relative homophily responds to changes in the meeting process.

Corollary 1. $I H_{1}(p)$ is shifted up by an increase in $\sigma$ or by an increase in $\gamma$.

That is, for a given society and homophily biases, decreasing the role of search-based meetings increases relative homophily. This results from the "dampening effect" of search-based meetings 
on network homophily. The more prevalent are search-based meetings in the formation process, the lower homophily will be, as "friends of friends" are less likely to be of the same type than are individuals met through the biased random meetings. Finally, increasing the location bias, all else equal, increases relative homophily for any value of $\sigma$, since this is the parameter that controls the extent to which random meetings are exogenously biased.

\section{Integration with biased search}

We now allow for the search part of the process to be biased as well as the random part. We do this for two main reasons. First, we want to assess what degree of integration is still compatible with this more flexible specification of biases in the network formation process. Second, the more realistic assumption of some form of bias also in the search process is needed to match the empirical patterns of scientific citations that we study in the final section of this paper.

To prepare for this more general version of the model, remember that in the analysis of Section 3, the random bias affects the choice of parents that are used to find the additional $\mathrm{nm}_{\mathrm{s}}$ search connections. In general, the bias that affects the search process may differ from, and possibly be a reinforcement of, the bias induced by the random process. This additional bias is described via an $H \times H$ matrix where each element is positive and of the form $B_{S}\left(\theta, \theta^{\prime}\right) .{ }^{15}$ A value of 1 indicates no additional bias, while a value greater (less) than 1 indicates a positive (negative) search bias of type $\theta$ towards type $\theta^{\prime}$. This is the distortion in the relative probabilities with which type $\theta$ searches the out-neighborhoods of its parent nodes.

The mean-field approximation of the process is described by

$$
P_{j}^{t+1}\left(\theta, \theta_{j}\right)=\frac{n m_{r}}{t} B_{r}\left(\theta, \theta_{j}\right)+\frac{m_{s}}{t} \sum_{\theta^{\prime}=1}^{H} B_{r}\left(\theta, \theta^{\prime}\right) B_{s}\left(\theta, \theta^{\prime}\right) \sum_{\lambda=j}^{t} P_{j}^{\lambda}\left(\theta^{\prime}, \theta_{j}\right) .
$$

The product $B_{r}\left(\theta, \theta^{\prime}\right) B_{S}\left(\theta, \theta^{\prime}\right)$ in (14) describes the probability applied by type $\theta$ to the selection of random nodes in search of type $\theta_{j}$. Note that the bias is independent of both time variables $j$ and $t$, and of the type $\theta_{j}$ of the target.

In matrix form, the system becomes

$$
\mathbf{P}_{t_{0}}^{t+1}=\frac{n m_{r}}{t} \mathbf{B}_{r}+\frac{m_{s}}{t}\left(\mathbf{B}_{s} \odot \mathbf{B}_{r}\right) \sum_{\lambda=t_{0}}^{t} \mathbf{P}_{t_{0}}^{\lambda},
$$

where $\odot$ is the Hadamard product: $\left(\mathbf{B}_{s} \odot \mathbf{B}_{r}\right)_{(i j)} \equiv \mathbf{B}_{s(i j)} \cdot \mathbf{B}_{r(i j)}$.

From the decomposition given in Eq. (2), it follows that

$$
\mathbf{B}_{s} \odot \mathbf{B}_{r}=\mathbf{B}_{s} \odot\left(\mathbf{Q} \mathbf{A} \mathbf{Q}^{-1}\right)=\mathbf{Q}\left(\mathbf{B}_{s} \odot \mathbf{A}\right) \mathbf{Q}^{-1},
$$

where the biases are such that $\mathbf{B}_{s} \odot \mathbf{A}$ is still a Markov matrix.

The model with unbiased search, analyzed in previous sections, is a special case of this model, where the matrix $\mathbf{B}_{s}$ is a matrix of all 1's.

As we will see, weak integration still holds in the presence of biased search. Moreover, while long-run integration generically does not hold, partial integration occurs under an additional

15 There are constraints on this bias matrix to have the resulting output be well-defined probabilities, but much can be deduced for general forms of the matrix, and so we only specify the (obvious) constraints as they become necessary. A more rigorous treatment and some explicit examples are in [11]. 
monotonicity condition. In this case, while the distortions in type frequencies among an individual's neighbors decrease over time, they do not vanish as the node accumulates links.

Proposition 9. If $m_{r}<1$, the general model with biased search satisfies the weak integration property, generically does not satisfy long-run integration, and satisfies the partial integration property provided that $\mathbf{B}_{s} \odot \mathbf{A}$ satisfies monotone convergence.

\section{An empirical application to citation data}

In this section we use our random-search model to study the patterns of cross-sub-field scientific citations in physics.

The use of scientific citation data is motivated by several factors. First, there is a literature that shows that key aspects of the time evolution of citations can be captured by models based on variations of a preferential attachment mechanism. [28], and then [31] for ISI papers and also [25], found that older papers enjoy an advantage in receiving citations, independently of the intrinsic quality of the paper. Although a bias in favor of recent papers allows for a better fit of certain datasets (see [3,35]), the evidence of a rich-gets-richer mechanism seems sound. In addition, Refs. $[34,36]$ have shown that this evidence can be accounted for when preferential attachment is generated by a random-search mechanism as the one we use in this paper, where authors first "randomly" (from the econometrician's perspective) select papers, and search these papers' reference lists to find additional citations.

There is less exploration of the patterns of citations across disciplines or across other types of categories in which research may be organized. Several works have shown that geographical distance and national boundaries are two important determinants of citation patterns, while [20] has shown that citation patterns are quite uniform across sub-fields in the high energy physics dataset (SPIRES). Also, [33] finds a relationship between the homophily in citing other papers and the total citations received by computer science papers.

Thus, the generative process of citations is consistent with basic aspects of the network formation process studied in this paper: First, it is a growing network process, since papers appear in chronological order, and old papers do not exit or die. Second, citations are directional, and only citations from newer to older nodes are possible. ${ }^{16}$ Third, citations cannot disappear, and accumulate over time. In addition, and specifically to our model, nodes have "types", that we identify with the scientific classification of a paper (see below for details). Finally, a key element of our process is that links are formed both at random and by search through established links. In the case of citations, these two channels of search are present, since there is a difference between citations that come from direct knowledge of a paper and citations that originate from the list of references of other papers that one has read.

We use the American Physical Society (APS) citations dataset, which reports papers published in journals of the APS between 1/1/1985 and 12/31/2003. ${ }^{17}$ There is a total of 207912 papers and 1488866 citations (roughly 7 citations per paper on average). Around 42.8 percent of the papers are never cited, while the most cited one receives 952 citations.

\footnotetext{
16 There are revisions of papers that allow them to cite relatively contemporaneous papers, but that seems to be a minor factor in the overall process.

17 The journals in our data are Physical Review A, B, C, D, E, Letters, STPER and RevModPhys; considering papers that have PACS classification codes which became compulsory in 1985 in the main six journals of the APS. This dataset is available online from http://prola.aps.org/, and contains the data analyzed in [29,30].
} 
Types are defined by the first digit of the first (out of at most four) PACS classification code that characterize each paper:

- 00: General;

- 10: The Physics of Elementary Particles and Fields;

- 20: Nuclear Physics;

- 30: Atomic and Molecular Physics;

- 40: Electromagnetism, Optics, Acoustics, Heat Transfer, Classical Mechanics, Fluid Dynamics;

- 50: Physics of Gases, Plasmas, and Electric Discharges;

- 60: Condensed Matter: Structural, Mechanical, and Thermal Properties;

- 70: Condensed Matter: Electronic Structure, Electrical, Magnetic, and Optical Properties;

- 80: Interdisciplinary Physics and Related Areas of Science and Technology;

- 90: Geophysics, Astronomy, and Astrophysics.

We first note that the time profiles of types' population shares, measured, for each type and for each year, as the proportion of the total papers published during that year that are of that given type, is fairly stationary during the whole period (see Fig. 1). ${ }^{18}$ The approximate stationarity of most categories is roughly in line with our assumption in the theoretical model that probabilities of birth of various types are time invariant. There are additional dramatic (unexplained) changes in the composition of fields following 2003, and so we truncate our data at that point.

In order to identify the various elements of our theoretical model, we need to distinguish citations that originate from a direct random draw from the pool of all existing papers ("random" citations) from those that originate from a search process that goes through the references contained in one's random citations ("search" citations). To do this, we proceed as follows. We first identify a citation from paper A to paper $\mathrm{C}$ as a "search" citation if there exists some paper B with the following properties: (1) B is published after C and before A, (2) A cites B, and (3) B cites C.

This method obviously has some degree of arbitrariness and will not perfectly identify how the authors found the papers they cite. The bias of this simplification is however not clear. On one side, it overstates the weight of "search" in the citation process, since A may well cite C because $\mathrm{C}$ is an important paper in the field, the reason for which also B also cites $\mathrm{C}$, without $\mathrm{A}$ having known about $\mathrm{C}$ though $\mathrm{B}$. On the other side, however, it could be that authors of paper A know about paper $\mathrm{C}$ only because they came across paper $\mathrm{B}$, which cites $\mathrm{C}$ : they could decide to cite only $\mathrm{C}$ because it contains an older version of the same idea. That is, it can be that some papers are found through the search process, without the authors ever citing the intermediate paper, and so some citations are coded as random even though they were found through

\footnotetext{
18 The only two sharp changes in the time profiles are around 1990 for type 10 (Physics of Elementary Particles and Fields) and type 70 (Condensed Matter Electronic Structure, Electrical, Magnetic, and Optical Properties). These changes were explained to us by the APS as follows (in private communication in response to our queries about these changes). The increase of type 70 was driven by the sharp increase in the subcategory 74 "Superconductivity", due to the spike in interest in High Temperature Superconductivity that began in 1986 (including some switching of fields of high energy particle physicists some of whom would have come from type 10). The sharp decrease of type 10 was due to a 1989 policy by the APS that increased page charges by 60 percent in the Physical Review journals, including Physical Review D where much elementary particle/high energy physics is published. Some authors reacted by publishing in other journals (outside of the APS data set) and increased use of the physics arXiv that started in 1991, and this reaction was particularly heavy in the particle physics community. In 1996 APS removed page charges for properly prepared electronic manuscripts in Physical Review C and D, and in 1999 did the same for the other Physical Review journals.
} 


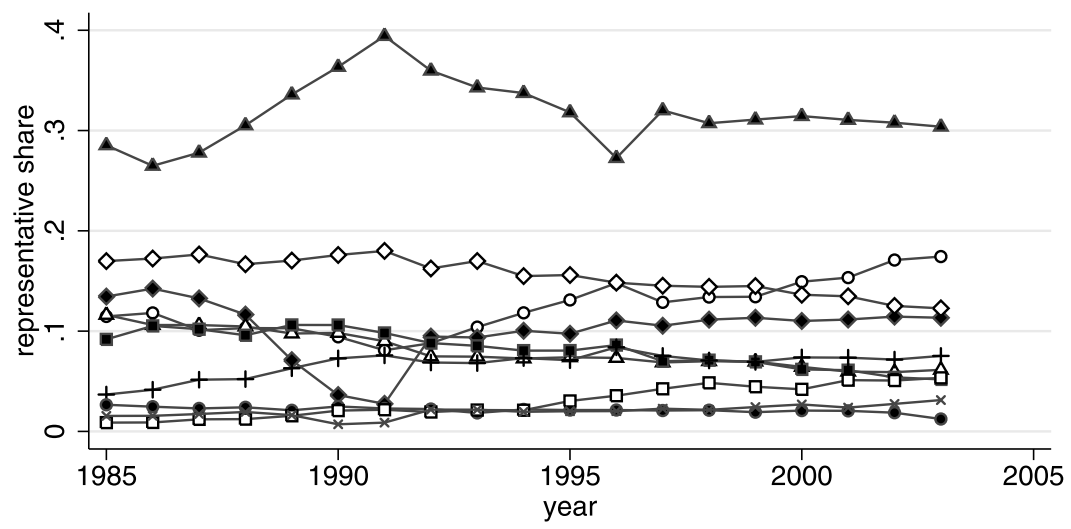

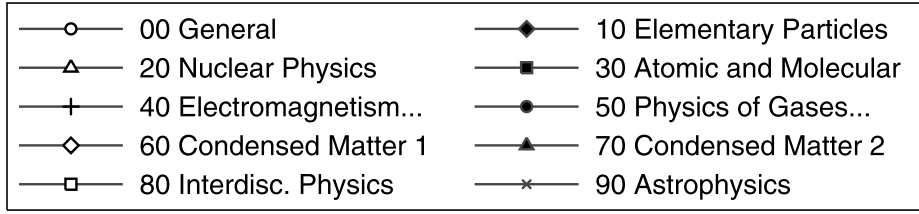

Fig. 1. Shares of types' proportions over time.

Table 1

Same-type bias in the "random" citations.

\begin{tabular}{lllllllllll}
\hline Classification & 00 & 10 & 20 & 30 & 40 & 50 & 60 & 70 & 80 & 90 \\
\hline Same type random cites: $q_{\text {out }}$ & 0.27 & 0.10 & 0.69 & 0.60 & 0.42 & 0.43 & 0.33 & 0.49 & 0.23 & 0.07 \\
Size of classification: $w$ & 0.13 & 0.10 & 0.08 & 0.08 & 0.07 & 0.02 & 0.15 & 0.32 & 0.03 & 0.02 \\
Coleman index & 0.17 & 0.00 & 0.67 & 0.57 & 0.37 & 0.42 & 0.21 & 0.26 & 0.20 & 0.05 \\
\hline
\end{tabular}

search. We stick with the strict interpretation of the model, given that we have no other way of identifying the actual process that the authors followed (see [36] for an interesting strategy of identification).

Using this method we identify 56.34 percent of total citations as "search" citations. We then classify the remaining 43.66 percent of citations as "random" citations, being the complement of the "search" citations.

\subsection{Bias in random out-citations}

In order to identify the bias in the random part of the process, we compare the share of "random" out-citations that are of the same type of the citing paper with the population share of the type of the citing paper. The first share $\left(q_{\text {out }}\right.$ in Table 1$)$ is obtained by averaging the share of random same-type out-citations of all papers of a given type during the whole time period. The second share ( $w$ in Table 1) is obtained as the share of papers of a given type over all papers in the sample for the whole time period.

The difference between these two shares is positive and substantial for all types, with maximum value of about 0.61 for type 20 , minimum value of 0.001 for type 10 , and average value 
of 0.26. Normalizing, for each type, this difference by the maximal potential difference given by one minus the population share of the type, we obtain the Coleman [8] homophily index of each type $\left(i h=\left(q_{\text {out }}-w\right) /(1-w)\right.$ in Table 1$) .{ }^{19}$ This index turns out not to be correlated with types' population shares.

\subsection{Search bias, long-run integration, and partial integration}

One challenge with an empirical investigation of the various concepts of integration is that certain papers happen to be intrinsically more cited than others, simply because they are more fundamental or important than others for their discipline. This type of "fitness" is independent of the age of the paper, and is not modeled in our analysis. ${ }^{20}$ More importantly, it could potentially outweigh the effect of time, and of the large in-degree that older nodes accumulate in time, which is one of the forces behind the long-run integration property.

We deal with this problem by looking at the type-composition of the first $\tau$ citations of each paper, thereby replacing time with citation order. This allows us to normalize the time-scale of each single paper, as if they all had the same fitness. In this new context, which takes a form similar to that of Proposition 5, the hypothesis we are testing is whether the homophily of the indegrees of a paper decreases with the order of its in-citations. This is meant to capture the main force that leads to partial integration: the growth of nodes' in-degree is to a large extent composed of in-citations of the "search" type, which in the case of unbiased search are less biased towards one's own type than in-citations of the "random" kind.

A way to test partial integration is to measure the probability that a given citation originated from a paper in the same field as the cited paper; more precisely, we estimate the change in this probability associated with an increase in the order of the in-citation. The partial integration hypothesis predicts that this probability should decrease with the order of the in-citation. We estimate a probit model where a dummy in-group (taking value one if the citation comes from a paper in the same field as the cited paper and zero otherwise) is estimated as a function of the order of the citation. The dataset contains 1034569 total citations, and we run separate regressions by looking at each sub-field separately (i.e., we look at all citations received by all papers belonging to each given sub-field). Results are reported in Table 2 .

We observe that for all sub-fields except type 00, type 50 and type 90, citations of higher order are less likely to come from papers within the same field. This is true also in the aggregate if we compute the expected change in probability without keeping track of the specific field of the receiving paper. Types 00 and 50 behave differently and have an increase in the probability of homophilous citations, while type 90 has no significant change in probability. At least broadly, this is consistent with partial integration. On average, the probability of being cited by papers of the same field decreases by $0.025 \%$ : on average, the share of in-field citations decreases by 2.5 percentage points between the first and the 100th in-citation.

Beyond testing partial integration, we also examine whether the fraction of a paper's citations from random versus search becomes more tilted towards search as its number of citations grows, as would be consistent with the model. This is plotted in Fig. 2 below.

There is an evident upward trend, consistent with the model's predictions.

19 This normalization has the purpose of allowing for meaningful comparison of groups of different sizes, by taking into account the maximal potential amount of homophily that each group has. See [9] for more discussion.

20 See [2] for the analysis of a single-type random-search process which is based on fitness. 
Table 2

Expected change in probability of a citation being homophilous associated with an increase in the order of the citation.

\begin{tabular}{lll}
\hline Type & \multicolumn{1}{l}{ DProb } & $P>|z|$ \\
\hline All fields & $-0.00025^{* *}$ & 0.000 \\
00 & $0.00028^{* *}$ & 0.000 \\
10 & $-0.00067^{* *}$ & 0.000 \\
20 & $-0.00191^{* *}$ & 0.000 \\
30 & $-0.00252^{* *}$ & 0.000 \\
40 & $-0.00034^{* *}$ & 0.000 \\
50 & $0.0008^{* *}$ & 0.009 \\
60 & $-0.00112^{* *}$ & 0.000 \\
70 & $-0.00024^{* *}$ & 0.000 \\
80 & $-0.00418^{* *}$ & 0.000 \\
90 & 0.00028 & 0.096 \\
\hline$* *$ & &
\end{tabular}

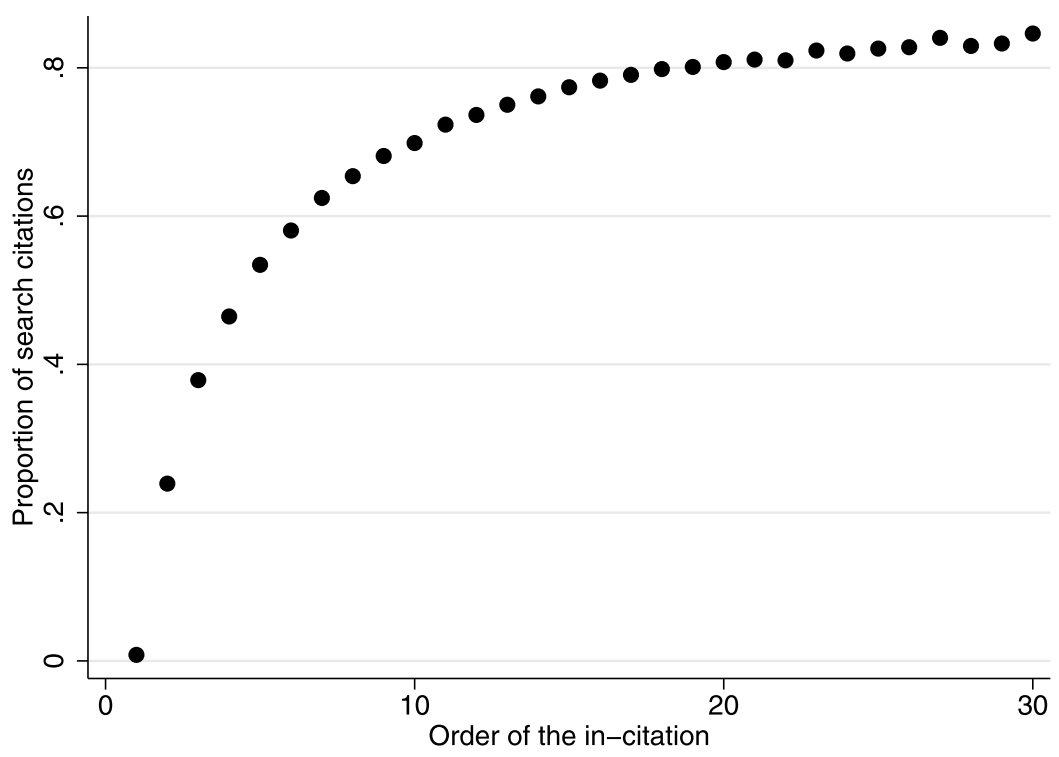

Fig. 2. Average fraction of search citations by citation order.

\section{Concluding remarks}

This paper contributes to our understanding of how heterogeneity and homophily among individuals impact the networks that they form. We have built on the framework of Jackson and Rogers [19], in which links result both from meeting others at random and through introductions to their neighbors, allowing both of these channels for link formation to be biased by the types of the nodes involved. Some applications of interest have significant type-based biases. Scholars are more likely to read papers from their own field, people are more likely to befriend those with a similar background, organizations have closer ties within departments, 
and so on. We do not attempt here to model the source of these biases, but take the model as a reduced form representation of the resulting effects that such biases have on how links are formed.

Within the context of this framework, long-run integration, whereby old nodes obtain local networks that asymptotically resemble the population at large, occurs if and only if the search part of the network formation process (the second channel mentioned above) is type-unbiased so that the only bias in the process comes in which nodes are initially found through the random meeting process.

Understanding the neighborhoods of old nodes is important since these nodes constitute the hubs of the network. If one is interested in processes occurring on the network such as, e.g., strategic behavior or diffusion processes, then the characteristics of hub nodes are of central importance. On the other hand, there are many other important properties of the network that may be affected by type-based biases. In order to analyze these properties, we turned to the more specific model of location-based biases among two groups, deriving implications regarding type-based degree distributions and on group-level homophily.

We leave open a number of interesting questions. First, there is the matter of exploring the extent to which the results from the location-based biases generalize. Second, there are other kinds of network formation processes in which similar questions could be addressed. In fact, in the different model of $[9,10]$, there are some similar results, but in general we have an incomplete understanding of how heterogeneity impacts network formation. Third, there are many summary statistics of networks that can be generalized to a multi-type framework, including clustering measures, that can be analyzed in future work.

\section{Appendix A. Some results on Markov matrices}

This first section of the appendix provides some results that are necessary for the proofs of our results. Take an $H \times H$ Markov matrix $\mathbf{M}$ with all positive elements, i.e. a positive Markov matrix.

Lemma 2. For every $x>0$ the $H \times H$ matrix

$$
\mathbf{M}(x) \equiv\left(e^{x}-1\right)^{-1} \sum_{\mu=1}^{\infty} \frac{x^{\mu}}{\mu !} \mathbf{M}^{\mu}=\frac{\exp (\mathbf{M} x)-\mathbf{I}}{\exp (x)-1}
$$

is a Markov matrix.

Proof. For every $\mu \in \mathbb{N}, \mathbf{M}^{\mu}$ is a Markov matrix. To show that $\mathbf{M}(x)$ is a Markov matrix, we need to prove that for every $i, j \in\{1, \ldots, H\}$ we have that $0<M(x)_{i j}<1$, and that $\sum_{k=1}^{H} M(x)_{k j}=1$.

The first condition comes from the fact that $M(x)_{i j}$ is a convex combination of (an infinite number of) probabilities.

The second condition comes from the fact that

$$
\sum_{k=1}^{H}[M(x)]_{k j}=\left(e^{x}-1\right)^{-1} \sum_{\mu=1}^{\infty} \frac{x^{\mu}}{\mu !}\left(\sum_{k=1}^{H}\left[M^{\mu}\right]_{k j}\right)=\left(e^{x}-1\right)^{-1} \sum_{\mu=1}^{\infty} \frac{x^{\mu}}{\mu !}=1 .
$$


$\mathbf{M}(x)$ can be seen as a weighted average of the infinite elements of $\left\{\mathbf{M}^{\mu}\right\}_{\mu \in \mathbb{N}}$. We know that

$$
\lim _{\mu \rightarrow \infty} \mathbf{M}^{\mu}=\left(\begin{array}{c}
\vec{v}(\mathbf{M})^{\prime} \\
\vdots \\
\vec{v}(\mathbf{M})^{\prime}
\end{array}\right),
$$

where the row-vector $\vec{v}(\mathbf{M})^{\prime}$ is the unique eigenvector associated with eigenvalue 1 of matrix $\mathbf{M}$ (up to a normalization that it's elements sum to one, by the Perron-Frobenius Theorem). We define this matrix at the limit, with all equal elements on each column, as $\overline{\mathbf{M}}$. Now we prove a relation between the limit of $\mathbf{M}(x)$ and $\overline{\mathbf{M}}$.

Lemma 3. For every positive Markov matrix $\mathbf{M}$, and for every couple $i, j \in\{1, \ldots, H\}$, we have that

$$
\lim _{x \rightarrow \infty}[M(x)]_{i j}=\lim _{\mu \rightarrow \infty}\left[M^{\mu}\right]_{i j}=[\bar{M}]_{i j} .
$$

Proof. By definition of $\overline{\mathbf{M}}$, for every $\epsilon>0$ there is a number $\bar{k} \in \mathbb{N}$, such that for every $\mu>\bar{k}$, we have $\left|\left[M^{\mu}\right]_{i j}-[M]_{i j}\right|<\epsilon$. By driving $x \rightarrow \infty$ we can impose to 0 the weight $\frac{x^{\nu}}{\left(e^{x}-1\right) v !}$ of every $v<\bar{k}$. In this way $[M(x)]_{i j}$ becomes a weighted average of almost only elements from $\left\{M^{\mu}\right\}_{\mu \in \mathbb{N}}$, with $\mu>\bar{k}$. As for all of them we have $\left|\left[M^{\mu}\right]_{i j}-[\bar{M}]_{i j}\right|<\epsilon$, we have the result.

Definition 4. $M$ satisfies the monotone convergence property if, for every couple $i, j \in$ $\{1, \ldots, H\}$, and for every $\mu \in \mathbb{N}$, the element $M_{i j}^{\mu}$ has the following properties:

1. if $M_{i j}>\bar{M}_{i j}$, then $M_{i j} \geqslant M_{i j}^{\mu} \geqslant M_{i j}^{\mu+1} \geqslant \bar{M}_{i j}$;

2. if $M_{i j}<\bar{M}_{i j}$, then $M_{i j} \leqslant M_{i j}^{\mu} \leqslant M_{i j}^{\mu+1} \leqslant \bar{M}_{i j}$.

What comes out directly from the definition is that, if $M_{i j}>\bar{M}_{i j}$, then there is at least one $\mu$ for which the inequality is strict, i.e. $M_{i j}^{\mu}>M_{i j}^{\mu+1}$.

Lemma 4. For every couple $i, j \in\{1, \ldots, H\}$, and for every $x>0$, if $\mathbf{M}$ satisfies the monotone convergence property, then

1. if $M_{i j}>\bar{M}_{i j}$, then $\frac{\partial}{\partial x}[M(x)]_{i j}<0$;

2. if $M_{i j}<\bar{M}_{i j}$, then $\frac{\partial}{\partial x}[M(x)]_{i j}>0$.

Proof. We focus on case 1 , as the other is proven by reversing inequalities.

First, note that the function

$$
\frac{\mu}{x}\left(e^{x}-1\right)-e^{x}
$$

is negative if and only if

$$
\mu<\frac{x e^{x}}{e^{x}-1} .
$$

Let us call $v(x)$ the minimum integer strictly above $\frac{x e^{x}}{e^{x}-1}$, i.e. $v(x) \equiv\left\lceil\frac{x e^{x}}{e^{x}-1}\right\rceil$. 
Now we can show that

$$
\begin{aligned}
\frac{\partial}{\partial x}[M(x)]_{i j} & =\frac{1}{\left(e^{x}-1\right)^{2}} \sum_{\mu=1}^{\infty} \frac{x^{\mu}}{\mu !}\left(\frac{\mu}{x}\left(e^{x}-1\right)-e^{x}\right)\left[M^{\mu}\right]_{i j} \\
& <\frac{1}{\left(e^{x}-1\right)^{2}} \sum_{\mu=1}^{\infty} \frac{x^{\mu}}{\mu !}\left(\frac{\mu}{x}\left(e^{x}-1\right)-e^{x}\right)\left[M^{v(x)}\right]_{i j} .
\end{aligned}
$$

It is a matter of calculus to check that

$$
\sum_{\mu=1}^{\infty} \frac{x^{\mu}}{\mu !}\left(\frac{\mu}{x}\left(e^{x}-1\right)-e^{x}\right)=0,
$$

and then the derivative in (17) is strictly negative.

Finally, we provide a simple sufficient condition for a $2 \times 2$ Markov matrix.

Lemma 5. Consider the $2 \times 2$ Markov matrix $\left(\begin{array}{cc}p & 1-p \\ 1-q & q\end{array}\right)$, if $p+q \geqslant 1$, then it has the monotone convergence property.

Proof. By the Perron-Frobenius Theorem this matrix converges to $\left(\begin{array}{ll}u & 1-u \\ u & 1-u\end{array}\right)$, with $u=$ $\frac{1-q}{(1-p)+(1-q)}$.

It is easy to check that, as $p \geqslant 1-q$, then $p \geqslant u$. By symmetry between $p$ and $q$, also $q \geqslant 1-u$.

Now consider a matrix $\left(\begin{array}{cc}\alpha & 1-\alpha \\ 1-\beta & \beta\end{array}\right)$, such that $p \geqslant \alpha \geqslant u$ and $q \geqslant \beta \geqslant 1-u$. To finish the proof it is enough to show that

$$
p \geqslant \alpha p+(1-\alpha)(1-q) \geqslant u,
$$

as it will be proved symmetrically also with respect to $q$ and $\beta$. The middle term of these inequalities is increasing in $\alpha$, as $p \geqslant 1-q$. If $\alpha=u$ it is equal to $u$, if instead $\alpha=p$, with some algebraic substitution, we have that again both inequalities are satisfied, as $p \geqslant 1-q$. This completes the proof.

\section{Appendix B. Proofs}

\section{B.1. Proofs for Section 3.2}

Proof of Proposition 1. (Page 9) Note first that the node born at time $t^{\prime}$ in Definition 1 has, at the beginning of time $t^{\prime}+1$ (before node $t^{\prime}+1$ sends its links) an in-degree of 0 . This directly implies that the probability of $t^{\prime}$ to receive a link at time $t^{\prime}+1$ from a node of type $\theta^{\prime \prime}$, given that such a node is born, is equal to the probability of being found at random among the $t^{\prime}$ nodes in the network. This probability is equal to

$$
\frac{n m_{r}}{t^{\prime}} p\left(\theta^{\prime \prime}\right)
$$

On the other hand, the probability that node $t_{0}$ is be found at time $t^{\prime}+1$ is the sum of the probability of being found at random and through search. In the model with homogeneous search, 
this is

$$
\frac{n m_{r}}{t^{\prime}} p\left(\theta^{\prime \prime}, \theta\left(t_{0}\right)\right)+n m_{s} \sum_{\theta \in \Theta} p\left(\theta^{\prime \prime}, \theta\right) \frac{\Pi_{t_{0}}^{t^{\prime}}\left(\theta, \theta\left(t_{0}\right)\right)}{t^{\prime} p\left(\theta\left(t_{0}\right)\right)} \frac{1}{n} .
$$

Note that in (20) the terms in the vector $\Pi_{t_{0}}^{t^{\prime}}\left(\theta, \theta\left(t_{0}\right)\right)$ grow without bound as $t^{\prime}$ tends to infinity, while the first terms in (20) and in (19) are constant once $t^{\prime}$ is eliminated from the denominator of both expressions. It follows that we can always choose a $t^{\prime}$ large enough for (20) to be larger than (19).

Proof of Proposition 2. (Page 10) We want to see how the matrix $\Pi_{t_{0}}^{t}$ of type-by-type links for a node born at time $t_{0}$ develops. To do this we compare its behavior with the behavior of the type-blind process, where the in-links evolve according to ${ }^{21}$

$$
\pi_{t_{0}}(t)=n \frac{m_{r}}{m_{s}}\left(\left(\frac{t}{t_{0}}\right)^{m_{s}}-1\right) .
$$

To make this comparison in the long run we study

$$
\lim _{t \rightarrow \infty} \frac{\Pi_{t_{0}}^{t}}{\pi_{t_{0}}(t)} .
$$

Consider the solution of the model, as described by Eq. (5), with the decomposition $\mathbf{B}_{r}=$ $\mathbf{Q A Q}^{-1}$.

We rewrite (5) as

$$
\Pi_{t_{0}}^{t}=\frac{m_{r}}{m_{s}}\left(\left(\frac{t}{t_{0}}\right)^{m_{s} \mathbf{Q A Q} \mathbf{Q}^{-1}}-\mathbf{I}\right) .
$$

We now use some results from Section 3.1. By Eq. (2), and the facts that $\mathbf{I}=\mathbf{Q I} \mathbf{Q}^{-1}$ and $\mathbf{A}^{n}=$ $\mathbf{Q} \mathbf{A}^{n} \mathbf{Q}^{-1}$, we obtain

$$
\begin{aligned}
\Pi_{t_{0}}^{t} & =n \frac{m_{r}}{m_{s}} \mathbf{Q}\left(\left(\sum_{\mu=0}^{\infty} \frac{\left(m_{s} \log \left(\frac{t}{t_{0}}\right) \mathbf{A}\right)^{\mu}}{\mu !}\right)-\mathbf{I}\right) \mathbf{Q}^{-1} \\
& =n \frac{m_{r}}{m_{s}}\left(\left(\frac{t}{t_{0}}\right)^{m_{s}}-1\right) \mathbf{Q}\left(\left(\left(\frac{t}{t_{0}}\right)^{m_{s}}-1\right)^{-1} \sum_{\mu=1}^{\infty} \frac{\left(m_{s} \log \left(\frac{t}{t_{0}}\right)\right)^{\mu}}{\mu !} \mathbf{A}^{\mu}\right) \mathbf{Q}^{-1} .
\end{aligned}
$$

Limit (21) implies that (we use Lemma 3 from Appendix A)

$$
\begin{aligned}
& \lim _{t \rightarrow \infty} \frac{\Pi_{t_{0}}^{t}}{\pi(t)}=\lim _{t \rightarrow \infty}\left(\mathbf{Q}\left(\left(\left(\frac{t}{t_{0}}\right)^{m_{s}}-1\right)^{-1} \sum_{\mu=1}^{\infty} \frac{\left(m_{s} \log \left(\frac{t}{t_{0}}\right)\right)^{\mu}}{\mu !} \mathbf{A}^{\mu}\right) \mathbf{Q}^{-1}\right) \\
& =\mathbf{Q}\left(\lim _{\mu \rightarrow \infty} \mathbf{A}^{\mu}\right) \mathbf{Q}^{-1} \\
& =\mathbf{Q}\left(\begin{array}{c}
\vec{v}(\mathbf{A})^{\prime} \\
\vdots \\
\vec{v}(\mathbf{A})^{\prime}
\end{array}\right) \mathbf{Q}^{-1}
\end{aligned}
$$

\footnotetext{
$\overline{21}$ This process reduces to the 1-type case studied in Jackson and Rogers [19].
} 
where the row-vector $\vec{v}(\mathbf{A})^{\prime}$ is the unique eigenvector associated with eigenvalue 1 of matrix $\mathbf{A}$ (normalized to sum to 1). In this way, in the long run a node of type $i$ born at time $t_{0}$ receives a fraction of in-links from nodes of type $j$ which is given by the ratio

$$
p(j) \frac{\vec{v}(\mathbf{A})_{i}}{p(i)}
$$

of the overall nodes that it would receive in a type-blind process. This proportion is the product of $p(j)$ times a term that is constant for type $i$.

Proof of Proposition 3. (Page 10) The result comes from the expression of matrix $\Pi_{t_{0}}^{t}$ as defined in Eq. (22), in the proof of Proposition 2:

$$
\frac{\Pi_{t_{0}}^{t}}{\pi_{t_{0}}(t)}=\mathbf{Q}\left(\left(\left(\frac{t}{t_{0}}\right)^{m_{s}}-1\right)^{-1} \sum_{\mu=1}^{\infty} \frac{\left(m_{s} \log \left(\frac{t}{t_{0}}\right)\right)^{\mu}}{\mu !} \mathbf{A}^{\mu}\right) \mathbf{Q}^{-1}
$$

Here $\left(\left(\frac{t}{t_{0}}\right)^{m_{s}}-1\right)^{-1}$ is just a rescaling term so that the matrix in brackets is again a Markov matrix (see Lemma 2 in Appendix A). From the proof of Proposition 2 we know that it converges to the distribution of the population shares. As A satisfies the monotone convergence property, we can apply Lemma 4 from Appendix A to prove that this convergence is monotonic.

Proof of Proposition 4. (Page 11) Expressing the steady-state equation (9) we obtain

$$
\overline{\mathbf{D}}=\left(1-m_{s}\right)\left(\mathbf{I}-m_{s} \mathbf{B}_{r}\right)^{-1} \mathbf{B}_{r} .
$$

Using the algebraic identity

$$
\left(\mathbf{I}-m_{s} \mathbf{B}_{r}\right)^{-1}=\sum_{\mu=0}^{\infty}\left(m_{s} \mathbf{B}_{r}\right)^{\mu}
$$

we obtain the following expression:

$$
\bar{D}=\mathbf{B}\left(\frac{1-m_{s}}{m_{s}} \sum_{\mu=1}^{\infty}\left(m_{s} \mathbf{A}\right)^{\mu}\right) \mathbf{B}^{-1} .
$$

In the above expression, the matrix in brackets is such that, as $m_{s} \rightarrow 1$, the elements of each column homogenize (see Lemma 3 of Appendix A). However, full homogeneity only occurs at the limit $m_{s} \rightarrow 1$.

To obtain some insight on the time evolution of the out-degree, let us express Eq. (8) as a differential equation, and solve it explicitly (as we have done in (5) for the in-degree).

The system is

$$
\frac{\partial}{\partial t} \boldsymbol{\Delta}_{t}=\left(1-m_{s}\right) \mathbf{B}_{r}+m_{s} \mathbf{B}_{r} \frac{\boldsymbol{\Delta}_{t}}{t},
$$

with solution:

$$
\boldsymbol{\Delta}_{t}=\overline{\mathbf{D}} t+\mathbf{C} t^{m_{s} \mathbf{B}_{r}},
$$

where $\mathbf{C}$ is a constant matrix.

For a given initial condition $\mathbf{D}_{1}$ (that we can identify with the matrix $\mathbf{A}$ of biases) the solution for $\mathbf{D}_{t}$ can be written as

$$
\mathbf{D}_{t}=\frac{\partial}{\partial t} \boldsymbol{\Delta}_{t}=\overline{\mathbf{D}}+\frac{1}{t}\left(\mathbf{D}_{1}-\overline{\mathbf{D}}\right) t^{m_{s} \mathbf{B}_{r}}
$$




\section{B.2. Proofs for Section 4}

For the proofs of this section we need an additional preliminary result, that follows here below.

Consider a degree distribution $F(k)$ obtained implicitly through a process such that the growth of a node born at $t_{0}$ is governed by $k_{t_{0}}(t)=f\left(t / t_{0}\right)$ and another degree distribution $G$ such that $k_{t_{0}}(t)=g\left(t / t_{0}\right)$, with $k_{t_{0}}\left(t_{0}\right)=0$. Assume $f$ and $g$ are weakly increasing and continuous on $\left[1,+\infty\left[\right.\right.$ and that $\lim _{x \rightarrow \infty} f(x)=\lim _{x \rightarrow \infty} g(x)=\infty$.

Lemma 6. $F$ first order stochastically dominates $G$ if and only if for all $x \geqslant 1, f(x) \geqslant g(x)$, with strict inequality for some $x$.

Proof. Assume $f(x) \geqslant g(x)$ for all $x \geqslant 1$. Pick $k$ and $t$ arbitrarily. Define $i_{f}$ as the birthdate of the node with degree $k$ at time $t$ under $f$, and similarly for $i_{g}$. We have $f\left(t / i_{f}\right)=k \geqslant g\left(t / i_{f}\right)$, which, since $g$ is non-decreasing implies that $i_{g} \leqslant i_{f}$. Since $F_{t}(k)=1-i_{f} / t$ and $G_{t}(k)=$ $1-i_{g} / t$, we have $F_{t}(k) \leqslant G_{t}(k)$.

Now take $\bar{x}$ such that $f(\bar{x})>g(\bar{x})$. Pick $k$ and $t$ arbitrarily. Define $i_{f}=t / \bar{x}$ and $\bar{k}$ to be the size of node $i_{f}$ at time $t$ under $f$. Then set $i_{g}$ to be the node with degree $\bar{k}$ at time $t$ under $g$. We have $\bar{k}=f\left(t / i_{f}\right)=f(\bar{x})>g(\bar{x})=g\left(t / i_{f}\right)$, which implies that $i_{g}<i_{f}$. Thus $F_{t}(\bar{k})<G_{t}(\bar{k})$.

To show necessity, fix $t$ and choose $i_{f}$ so that $f\left(t / i_{f}\right)<g\left(t / i_{f}\right)$, and set $\bar{k}=f\left(t / i_{f}\right)$. Defining $i_{g}$ as the node with degree $\bar{k}$ at time $t$ under $f$, we know that $i_{g}>i_{f}$. This implies that $G_{t}(\bar{k})<F_{t}(\bar{k})$, completing the proof.

Now we can proceed with the proofs of Section 4.

Proof of Lemma 1. (Page 13) Apply to this particular case the expression from (5), considering the decomposition from (2):

$$
\Pi_{t_{0}}^{t}=n \frac{m_{r}}{m_{s}}\left(\mathbf{Q}\left(\frac{t}{t_{0}}\right)^{m_{s}\left(\begin{array}{cc}
p_{1} & 1-p_{1} \\
1-p_{2} & p_{2}
\end{array}\right)} \mathbf{Q}^{-1}-\mathbf{I}\right),
$$

where we have called $p_{1} \equiv p\left(\theta_{1}, \theta_{1}\right)$ and $p_{2} \equiv p\left(\theta_{2}, \theta_{2}\right)$.

As can be directly computed checking [22], we have that

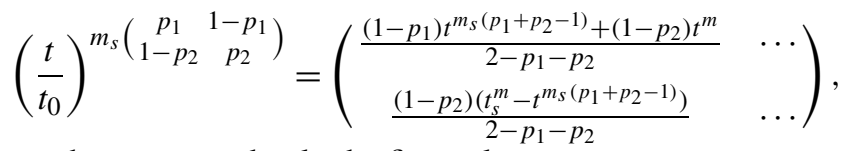

where we have reported only the first column.

Recall that $p_{1}=\frac{p \gamma^{2}}{(1-p)(1-\gamma)+p \gamma}+\frac{p(1-\gamma)^{2}}{p(1-\gamma)+(1-p) \gamma}$ and $p_{2}=\frac{(1-p) \gamma^{2}}{p(1-\gamma)+(1-p) \gamma}+\frac{(1-p)(1-\gamma)^{2}}{(1-p)(1-\gamma)+p \gamma}$. Some manipulations show that $\frac{1-p_{1}}{2-p_{1}-p_{2}}=1-p$, and symmetrically $\frac{1-p_{2}}{2-p_{1}-p_{2}}=p .^{22}$ If we finally consider that $\mathbf{Q}=\left(\begin{array}{cc}p & 0 \\ 0 & 1-p\end{array}\right)$, we have the result.

Proof of Proposition 5. (Page 13) We take the case of $\theta_{1}$. The case of $\theta_{2}$ is analogous. Define $f(x)=n m_{r} / m_{s}\left(x^{m_{s}}-1\right)$; hence $f^{-1}(k)=\left(1+\frac{k m_{s}}{n m_{r}}\right)^{1 / m_{s}}$. Next define $g(x)=p+$

$22\left(\frac{1-p_{1}}{2-p_{1}-p_{2}}, \frac{1-p_{2}}{2-p_{1}-p_{2}}\right)$ is actually the eigenvector of $\left(\begin{array}{cc}p_{1} & 1-p_{1} \\ 1-p_{2} & p_{2}\end{array}\right)$ associated to its asymptotic limit $\lim _{t \rightarrow \infty}\left(\begin{array}{cc}p_{1} & 1-p_{1} \\ 1-p_{2} & p_{2}\end{array}\right)^{t}$ (see the proof of Lemma 5 in Appendix A). Considering the location-based model, it is reasonable that this limit does not depend on $\gamma$ but only on the initial distribution of the two types, given by $p$. 
$(1-p)\left(x^{b m_{s}}-1\right) /\left(x^{m_{s}}-1\right)$. Notice that at time $t$, the proportion of in-links that a node of type $\theta_{1}$ born at time $t_{0}<t$ has from its own group is

$$
\frac{\Pi_{t_{0}}^{t}(1,1)}{\Pi_{t_{0}}^{t}(1,1)+\Pi_{t_{0}}^{t}(2,1)}
$$

It then follows from Lemma 1 that $r^{1}(k)=g\left(f^{-1}(k)\right)$. Evaluating this formula delivers the claimed expression.

Without loss of generality, we can set $n m_{r} / m_{s}=1$ in what follows. Introduce $y=1+k$. Next, $r^{\prime \prime}=\left[\left(f^{-1}\right)^{\prime}\right]^{2} g^{\prime \prime} \circ f^{-1}+\left(f^{-1}\right)^{\prime \prime} g^{\prime} \circ f^{-1}$. Developing and substituting shows that $r^{\prime \prime}$ has the same sign as

$$
\varphi(y)=y^{b+2}(1-b)(2-b)+y^{b+1} 2 b(2-b)-y^{b} b(1-b)-2 y^{2} .
$$

A detailed study of $\varphi$ and its first three derivatives then shows that $\varphi(y)>0$ if $y>1$, hence that $r^{\prime \prime}(k)>0$ if $k>0$.

The explicit expressions for the derivative with respect to $p$ are not trivial given that $b$ is a function of $p$. We have

$$
\begin{aligned}
\frac{\partial r}{\partial k}=-(1-p) \frac{(1+k)^{b-1}(1+(1-b) k)-1}{k^{2}}, & \\
k^{2}(1+k)^{1-b} \frac{\partial^{2} r}{\partial k \partial p}= & \psi(k)=1+(1-b) k-(1+k)^{1-b} \\
& +(1-p)\left(-\frac{\partial b}{\partial p}\right)[\ln (1+k)(1+(1-b) k)-k] .
\end{aligned}
$$

Also, note that $r(0)=p+(1-p) b$. Thus, $\frac{\partial r}{\partial p}(0)=1-b+\frac{\partial b}{\partial p}(1-p)$. We have $b=1-$ $\frac{\gamma(1-\gamma)}{p(1-p)(2 \gamma-1)^{2}+\gamma(1-\gamma)}$ and $\frac{\partial b}{\partial p}=-\frac{(2 p-1)(2 \gamma-1)^{2} \gamma(1-\gamma)}{\left[p(1-p)(2 \gamma-1)^{2}+\gamma(1-\gamma)\right]^{2}}$. Developing, we get that $\frac{\partial r}{\partial p}(0)$ has the same sign as $1-(2 \gamma-1)^{2} p(2-p)-3 \gamma(1-\gamma) \geqslant 1-(2 \gamma-1)^{2}-3 \gamma(1-\gamma)=\gamma(1-\gamma)>0$ where the first inequality comes from the fact that $p(2-p) \leqslant 1$. Thus, $\frac{\partial r}{\partial p}(0)>0$ and $1-b>$ $\left(-\frac{\partial b}{\partial p}\right)(1-p)$.

Next, derive the function $\psi$ with respect to $k$. We have

$$
\psi^{\prime}(k)=(1-b)\left(1-(1+k)^{-b}\right)+(1-p)\left(-\frac{\partial b}{\partial p}\right)\left[(1-b) \ln (1+k)-b\left(1-\frac{1}{1+k}\right)\right]
$$

and

$$
\begin{aligned}
(1+k) \psi^{\prime \prime}(k)= & (1-p)\left(-\frac{\partial b}{\partial p}\right)(1-b)-(1-p)\left(-\frac{\partial b}{\partial p}\right) b(1+k)^{-1} \\
& +b(1-b)(1+k)^{-b} .
\end{aligned}
$$

Here, $\psi^{\prime \prime}(0)=b(1-b)+(1-p)\left(-\frac{\partial b}{\partial p}\right)(1-2 b)$. Since $1-b>\left(-\frac{\partial b}{\partial p}\right)(1-p)$, we have $\psi^{\prime \prime}(0) \geqslant(1-p)\left(-\frac{\partial b}{\partial p}\right)(1-b)>0$. Also, $\lim _{k \rightarrow \infty} \psi^{\prime \prime}(k)=0^{+}$. By looking at its derivative, we see that the function $(1+k) \psi^{\prime \prime}(k)$ is either decreasing, or increasing and then decreasing. In either case, since it is positive when $k=0$ and when $k \rightarrow \infty$, it must be greater than or equal to zero for any $k$. Thus, $\psi^{\prime \prime}>0$ if $k>0$ hence $\psi^{\prime}$ is increasing. Since $\psi^{\prime}(0)=0, \psi^{\prime}>0$ if $k>0$. Thus, $\psi$ is increasing and as $\psi(0)=0, \psi>0$ and $\frac{\partial^{2} r}{\partial k \partial p}>0$ if $k>0$. Finally, given that $\frac{\partial r}{\partial p}(0)>0$ and $\frac{\partial r}{\partial p}$ is increasing in $k, \frac{\partial r}{\partial p}>0, \forall k$. 
Proof of Proposition 6. (Page 14) For (i), use Lemma 1 to write

$$
\begin{aligned}
& \Pi_{t_{0}}^{t}(1,1)=n \frac{m_{r}}{m_{s}} p\left[\left(\frac{t}{t_{0}}\right)^{m_{s}}-\left(\frac{t}{t_{0}}\right)^{b m_{s}}\right]+n \frac{m_{r}}{m_{s}}\left[\left(\frac{t}{t_{0}}\right)^{b m_{s}}-1\right], \\
& \Pi_{t_{0}}^{t}(2,1)=n \frac{m_{r}}{m_{s}}(1-p)\left[\left(\frac{t}{t_{0}}\right)^{m_{s}}-\left(\frac{t}{t_{0}}\right)^{b m_{s}}\right] .
\end{aligned}
$$

Given that $p>1 / 2$ and that $b \geqslant 0$, we know that $\frac{1-p}{p}<1$ and the second term in the first equation is non-negative. Thus $\Pi_{t_{0}}^{t}(1,1)>\Pi_{t_{0}}^{t}(2,1)$ for all $t \geqslant t_{0}$, which allows us to apply Lemma 6.

Now consider the expressions for $\Pi_{t_{0}}^{t}(2,2)$ and $\Pi_{t_{0}}^{t}(1,2)$ obtained from the above equations by exchanging $p$ with $1-p$. When $p>1 / 2$ (meaning $\theta_{1}$ is the majority group) then $\frac{p}{1-p}>1$, and when $b<1$ (i.e., $\gamma<1$, meaning there is at least some inter-group linking) then for large values of $t / t_{0}$ the second term in the expression for $\Pi_{t_{0}}^{t}(2,2)$ becomes negligible, in which case $\Pi_{t_{0}}^{t}(2,2)<\Pi_{t_{0}}^{t}(1,2)$ in the upper tail, proving (ii) by application of Lemma 6.

For (iii), introduce the function $\psi(x)=p(1-p)\left[x^{m_{s}}-x^{b m_{s}}\right]-(1-p)\left[(1-p) x^{m_{s}}+\right.$ $\left.p x^{b m_{s}}-1\right]$. Note that $\psi\left(t / t_{0}\right) \geqslant 0$ if and only if $\Pi_{t_{0}}^{t}(1,2) \geqslant \Pi_{t_{0}}^{t}(2,2)$. Observe that $\psi(1)=0$. Also,

$$
\psi^{\prime}(x)=x^{m_{s}-1}\left[(2 p-1)(1-p) m_{s}-2 p(1-p) b m_{s} x^{(b-1) m_{s}}\right] .
$$

Since $b-1 \leqslant 0$, the second term of the RHS is weakly increasing in $x$. There are two cases. First, $\psi^{\prime}(1) \geqslant 0$, in which case $\forall x \geqslant 1, \psi^{\prime}(x) \geqslant 0$, thus $\psi$ is weakly increasing and $\forall x \geqslant 1$, $\psi(x) \geqslant 0$. Otherwise $\psi^{\prime}(1)<0$, in which case $\psi^{\prime}$ is first negative then positive above 1 (since $\left.\psi^{\prime}(\infty)=\infty\right)$, hence $\psi$ is first decreasing and then increasing, which also means that $\psi$ is first negative and then positive above 1 . Therefore, $F_{21}$ FOSD $F_{22}$ if and only if $\psi^{\prime}(1) \geqslant 0$. The condition reduces to

$$
b \leqslant \frac{2 p-1}{2 p} .
$$

For (iv), working under the original model, previous equations reduce to $\Pi_{t_{0}}^{t}(1,1)=$ $\left(n m_{r} / m_{s}\right)\left[p\left(\frac{t}{t_{0}}\right)^{m_{s}}+(1-p)\left(\frac{t}{t_{0}}\right)^{b m_{s}}-1\right], \Pi_{t_{0}}^{t}(2,1)=\left(n m_{r} / m_{s}\right)(1-p)\left[\left(\frac{t}{t_{0}}\right)^{m_{s}}-\left(\frac{t}{t_{0}}\right)^{b m_{s}}\right]$ and $\Pi_{t_{0}}^{t}(1,1)+\Pi_{t_{0}}^{t}(2,1)=\left(n m_{r} / m_{s}\right)\left[\left(\frac{t}{t_{0}}\right)^{m_{s}}-1\right]$, which does not depend on $p$. This proves the result.

Proof of Proposition 7. (Page 15) Observe that $b$ increases with $\gamma$. This means that $\left(\Pi_{t_{0}}^{t}\right)^{\prime}(1,1) \geqslant \Pi_{t_{0}}^{t}(1,1)$ and $\left(\Pi_{t_{0}}^{t}\right)^{\prime}(2,2) \geqslant \Pi_{t_{0}}^{t}(2,2)$ while $\left(\Pi_{t_{0}}^{t}\right)^{\prime}(2,1) \leqslant \Pi_{t_{0}}^{t}(2,1)$ and $\left(\Pi_{t_{0}}^{t}\right)^{\prime}(1,2) \leqslant \Pi_{t_{0}}^{t}(1,2)$. The result then follows from Lemma 6.

Proof of Proposition 8. (Page 16) Substituting from Eq. (10), we have

$$
I H_{1}(p)=\frac{(1-2 \gamma)^{2} \sigma p(1-p)}{\sigma p(1-p)+\left(1+(1-2 p)^{2} \sigma\right) \gamma(1-\gamma)} .
$$

From this expression, it is easily verified that $I H_{1}(p)=I H_{1}(1-p)$ and that $I H_{1}(0)=$ $I H_{1}(1)=0$. The first derivative of $I H_{1}$ is

$$
\frac{\partial I H_{1}(p)}{\partial p}=\frac{(1-2 p) \sigma(\sigma+1)(2 \gamma-1)^{2} \gamma(1-\gamma)}{\left((\sigma+1) \gamma(1-\gamma)+(2 \gamma-1)^{2} p \sigma(1-p)\right)^{2}},
$$


which has the same sign as $1-2 p$, proving that $I H_{1}$ is increasing below $p=1 / 2$ and then decreasing. To show concavity, write the second derivative as

$$
\begin{aligned}
& \frac{\partial^{2} I H_{1}(p)}{\partial p^{2}} \\
& \quad=\frac{2 \gamma(1-\gamma)(2 \gamma-1)^{2} \sigma(\sigma+1) *\left(\sigma\left(3 p^{2}-3 p+1\right)-\gamma(1-\gamma)\left(3 \sigma(2 p-1)^{2}-1\right)\right)}{-\sigma^{3}\left(\gamma(1-\gamma)\left((2 p-1)^{2}+1\right)+p(1-p)\right)^{3}} .
\end{aligned}
$$

The denominator is negative, and the term in the numerator before the asterisk is positive, so $\mathrm{IH}_{1}$ is concave if and only if $\sigma\left(3 p^{2}-3 p+1\right)-\gamma(1-\gamma)\left(3 \sigma(2 p-1)^{2}-1\right)>0$. Dividing by $\sigma$ and rearranging, we must show that $\gamma(1-\gamma)\left(3(2 p-1)^{2}-1 / \sigma\right)+3 p(1-p)<1 . \gamma(1-\gamma) \leqslant 1 / 4$ and $-1 / \sigma \leqslant 0$; using these inequalities and collecting terms proves the result.

Proof of Corollary 1. (Page 16) The relevant derivatives are

$$
\begin{aligned}
\frac{\partial I H_{1}}{\partial \sigma} & =\frac{(1-2 \gamma)^{2} \gamma(1-\gamma) p(1-p)}{\left(\sigma p(1-p)+\gamma(1-\gamma)\left(1+(1-2 p)^{2}\right) \sigma\right)^{2}}, \\
\frac{\partial I H_{1}}{\partial \gamma} & =\frac{(2 \gamma-1) p(1-p) \sigma(1+\sigma)}{\left(\sigma p(1-p)+\gamma(1-\gamma)\left(1+(1-2 p)^{2}\right) \sigma\right)^{2}},
\end{aligned}
$$

both of which are easily verified as being positive.

\section{B.3. Proofs for Section 5}

Proof of Proposition 9. (Page 18) For what concerns the weak integration property, see the proof of Proposition 1. The only thing to change is the right-hand part of Eq. (14) instead of the formula in (20).

For long-run integration, consider the solution to the general model, with biased search, as described by Eq. (15). We follow the same procedure as in the proof of Proposition 2, since $\mathbf{B}_{s} \odot \mathbf{A}$ is still a Markov matrix. We obtain

$$
\lim _{t \rightarrow \infty} \frac{\Pi_{t_{0}}^{t}}{\pi_{t_{0}}(t)}=\left(\mathbf{B}_{s} \odot \mathbf{B}_{r}\right)^{-1} \mathbf{B}_{r} \mathbf{Q}\left(\begin{array}{c}
\vec{v}\left(\mathbf{B}_{s} \odot \mathbf{A}\right)^{\prime} \\
\vdots \\
\vec{v}\left(\mathbf{B}_{s} \odot \mathbf{A}\right)^{\prime}
\end{array}\right) \mathbf{Q}^{-1} .
$$

In the long run a node of type $i$ born at time $t_{0}$ will receive a number of in-links from nodes of type $j$ which is a fraction

$$
\begin{aligned}
{\left[\lim _{t \rightarrow \infty} \frac{\Pi_{t_{0}}^{t}}{\pi_{t_{0}}(t)}\right]_{i j} } & =\sum_{h=1}^{H}\left(\left[\left(\mathbf{B}_{s} \odot \mathbf{B}_{r}\right)^{-1} \mathbf{B}_{r}\right]_{j h} p(h) \frac{\vec{v}\left(\mathbf{B}_{s} \odot \mathbf{A}\right)_{i}}{p(i)}\right) \\
& =\sum_{h=1}^{H}\left(\sum_{k=1}^{H}\left(\left[\left(\mathbf{B}_{s} \odot \mathbf{B}_{r}\right)^{-1}\right]_{j k}\left[\mathbf{B}_{r}\right]_{k h}\right) p(h) \frac{\vec{v}\left(\mathbf{B}_{s} \odot \mathbf{A}\right)_{i}}{p(i)}\right) \\
& =\sum_{h=1}^{H}\left(\sum_{k=1}^{H}\left(\left[\left(\mathbf{B}_{s} \odot \mathbf{B}_{r}\right)^{-1}\right]_{j k} p(k) \frac{p(k, h)}{p(h)}\right) p(h) \frac{\vec{v}\left(\mathbf{B}_{s} \odot \mathbf{A}\right)_{i}}{p(i)}\right)
\end{aligned}
$$




$$
\begin{aligned}
& =\left(\sum_{h=1}^{H} \sum_{k=1}^{H}\left(\left[\left(\mathbf{B}_{s} \odot \mathbf{B}_{r}\right)^{-1}\right]_{j k} p(k) p(k, h)\right)\right) \frac{\vec{v}\left(\mathbf{B}_{s} \odot \mathbf{A}\right)_{i}}{p(i)} \\
& =\left(\sum_{k=1}^{H}\left[\left(\mathbf{B}_{s} \odot \mathbf{B}_{r}\right)^{-1}\right]_{j k} p(k)\right) p(j) \frac{\vec{v}\left(\mathbf{B}_{s} \odot \mathbf{A}\right)_{i}}{p(i)}
\end{aligned}
$$

of the overall links that it would receive in a type-blind process, where the last line comes from the fact that $\sum_{h=1}^{H} p(k, h)=1$. The second term is still a constant for type $i$, but the first term is generically not proportional to $p(j)$.

Finally, for the partial integration property, the proof is analogous to the proof of Proposition 3. In this case

$$
\frac{\Pi_{t_{0}}^{t}}{\pi_{t_{0}}(t)}=\left(\mathbf{B}_{s} \odot \mathbf{B}_{r}\right)^{-1} \mathbf{B}_{r} \mathbf{Q}\left(\left(\left(\frac{t}{t_{0}}\right)^{m_{s}}-1\right)^{-1} \sum_{\mu=1}^{\infty} \frac{\left(m_{s} \log \left(\frac{t}{t_{0}}\right)\right)^{\mu}}{\mu !}\left(\mathbf{B}_{s} \odot \mathbf{A}\right)^{\mu}\right) \mathbf{Q}^{-1} .
$$

As $\mathbf{B}_{s} \odot \mathbf{A}$ satisfies the monotone convergence property, we can use Lemma 4 from Appendix A, and the same reasoning applies.

\section{References}

[1] R. Albert, A.-L. Barabási, Emerging of scaling in random networks, Science 286 (1999) 509-512.

[2] E. Atalay, Sources of variation in social networks, Department of Economics, University of Chicago, Mimeo, 2011.

[3] K. Börner, J.T. Maru, R.L. Goldstone, The simultaneous evolution of author and paper networks, Proc. Natl. Acad. Sci. 101 (2004) 5266-5273.

[4] Y. Bramoullé, B. Rogers, Diversity and popularity in social networks, Northwestern University and Université Laval, Mimeo, 2010.

[5] S. Breschi, F. Lissoni, Mobility of inventors and the geography of knowledge spillovers. New evidence on US data, CESPRI WP n. 184, 2006.

[6] F. Chung, L. Lu, The average distances in random graphs with given expected degrees, Proc. Natl. Acad. Sci. USA 99 (2002) 15879-15882.

[7] F. Chung, L. Lu, Connected components in random graphs with given degree sequences, Ann. Comb. 6 (2002) $125-145$.

[8] J. Coleman, Relational analysis: the study of social organizations with survey methods, Human Organ. 17 (1958) 28-36.

[9] S. Currarini, M.O. Jackson, P. Pin, An economic model of friendship: Homophily, minorities and segregation, Econometrica 77 (2009) 1003-1045.

[10] S. Currarini, M.O. Jackson, P. Pin, Identifying the roles of choice and chance in network formation: Racial biases in high school friendships, Proc. Natl. Acad. Sci. 107 (2010) 4857-4861.

[11] S. Currarini, M.O. Jackson, P. Pin, Long-run integration in social networks, Stanford University, Mimeo, 2010.

[12] B. Golub, M.O. Jackson, How Homophily Affects the Speed of Learning and Best Response Dynamics, Quart. J. Econ. (2009), forthcoming.

[13] L.A. Kóczy, A. Nichifor, M. Strobel, Intellectual influence: Quality versus quantity, Maastricht Research School of Economics of Technology and Organization, Mimeo, 2010.

[14] A.B. Jaffe, M. Trajtenberg, Flows of knowledge from universities and federal laboratories: Modeling the flow of patent citations over time and across institutional and geographic boundaries, Proc. Natl. Acad. Sci. USA 93 (1996) 12671-12677.

[15] M.O. Jackson, Social structure, segregation, and economic behavior, presented as the Nancy Schwartz Memorial Lecture, 2007.

[16] M.O. Jackson, Average distance, diameter, and clustering in social networks with homophily, in: C. Papadimitriou, S. Zhang (Eds.), Proceedings of the Workshop in Internet and Network Economics (WINE 2008), in: Lecture Notes in Comput. Sci., Springer-Verlag, Berlin, Heidelberg, 2008.

[17] M.O. Jackson, Social and Economic Networks, Princeton University Press, 2008. 
[18] M.O. Jackson, D. Lopez-Pintado, Diffusion in networks with heterogeneous agents and homophily, Mimeo, http:// ssrn.com/abstract=1950476, 2011.

[19] M.O. Jackson, B. Rogers, Meeting strangers and friends of friends: How random are social networks?, Amer. Econ. Rev. 97 (2007) 890-915.

[20] S. Lehmann, B. Lautrup, A.D. Jackson, Citation networks in high energy physics, Phys. Rev. E 68 (2004) 026113.

[21] M. McPherson, L. Smith-Lovin, J.M. Cook, Birds of a feather: Homophily in social networks, Annu. Rev. Sociol. 27 (2001) 415-444.

[22] I. Moiseevich Ryzhik, A. Jeffrey, D. Zwillinger, Table of Integrals, Series and Products, Elsevier, 2007.

[23] M. Newman, The structure and function of complex networks, SIAM Rev. 45 (2003) 167-256.

[24] M. Newman, Coauthorship networks and patterns of scientific collaboration, Proc. Natl. Acad. Sci. USA 101 (2004) 5200-5205.

[25] M. Newman, First-mover advantage in scientific publication, Europhys. Lett. 86 (2009) 68001.

[26] I. Palacios-Huerta, O. Volij, The measurement of intellectual influence, Econometrica 72 (2004) 963-977.

[27] D.J.S. Price, Networks of scientific papers, Science 149 (1965) 510-515.

[28] D.J.S. Price, A general theory of bibliometric and other cumulative advantage processes, J. Am. Soc. Inf. Sci. 27 (1976) 292-306.

[29] F. Radicchi, C. Castellano, Rescaling citations of publications in physics, Phys. Rev. E 83 (2011) 46-116.

[30] F. Radicchi, S. Fortunato, B. Markines, A. Vespignani, Diffusion of scientific credits and the ranking of scientists, Phys. Rev. E 80 (2009) 56-103.

[31] S. Redner, How popular is your paper? An empirical study of the citation distribution, Eur. Phys. J. B 4 (1998) 131-134.

[32] E.J. Rinia, T.N. van Leeuwen, E.E.W. Bruins, H.G. van Vuren, A.F.J. van Raan, Citation delay in interdisciplinary knowledge exchange, Scientometrics 51 (2001) 293-309.

[33] X. Shi, B. Tseng, L. Adamic, Information diffusion in computer science citation networks, in: Proceedings of the Third International ICWSM Conference, 2009.

[34] M.V. Simkin, V.P. Roychowdhury, Stochastic modeling of citation slips, Scientometrics 62 (2005) 367-384.

[35] M.V. Simkin, V.P. Roychowdhury, A mathematical theory of citing, J. Am. Soc. Inf. Sci. 58 (2007) 1661-1673.

[36] M.V. Simkin, V.P. Roychowdhury, Theory of citing, Department of Electrical Engineering, University of California, Los Angeles, Mimeo, 2011.

[37] D.J. Watts, S.H. Strogatz, Collective dynamics of 'small-world' networks, Nature 393 (1998) 440-442. 